\title{
Equivalent Realisations of a Rigid Graph
}

\author{
Bill Jackson * J.C. Owen ${ }^{\dagger}$ \\ 2 December, 2017
}

\begin{abstract}
Given a rigid realisation of a graph $G$ in $\mathbb{R}^{2}$, it is an open problem to determine the maximum number of pairwise non-congruent realisations which have the same edge lengths as the given realisation. This problem can be restated as finding the number of solutions of a related system of quadratic equations and in this context it is natural to consider the number of solutions in $\mathbb{C}^{2}$ rather that $\mathbb{R}^{2}$. We show that the number of complex solutions, $c(G)$, is the same for all generic realisations of a rigid graph $G$, characterise the graphs $G$ for which $c(G)=1$, and show that the problem of determining $c(G)$ can be reduced to the case when $G$ is 3-connected and has no non-trivial 3 -edge-cuts. We obtain results on the effect of the Henneberg moves and the vertex-splitting operation on $c(G)$. We then use these to determine $c(G)$ exactly for two important families of graphs, and show that the graphs in both families have $c(G)$ pairwise equivalent generic real realisations. We also show that every planar isostatic graph on $n$ vertices has at least $2^{n-3}$ pairwise equivalent generic real realisations.
\end{abstract}

Keywords: rigid framework; number of equivalent realisations

\section{Introduction}

Graphs with geometrical constraints provide natural models for a variety of applications, including Computer-Aided Design, sensor networks and flexibil-

*School of Mathematical Sciences, Queen Mary University of London, Mile End Road, London E1 4NS, England. E-mail: b.jackson@qmul.ac.uk

†Siemens, Park House, Cambridge CB3 0DU, England. E-mail: owen.john.ext@siemens.com 
ity in molecules. Given a graph $G$ and prescribed lengths for its edges, a basic problem is to determine whether $G$ has a straight line realisation in Euclidean $d$-dimensional space with these given lengths. Closely related problems are to determine whether a given realisation is unique or, more generally, determine how many distinct realisations exist with the same edge lengths. Saxe [30] has shown that both the existence and uniqueness problems are NP-hard. However, this hardness relies on algebraic relations between coordinates of vertices, and for practical purposes it is natural to study generic realisations.

Gortler, Healy and Thurston [12] showed that the uniqueness of a generic realisation in $\mathbb{R}^{d}$ depends only on the structure of the underlying graph, and we say that a graph $G$ is globally rigid in $\mathbb{R}^{d}$ if it has a unique generic realisation in $\mathbb{R}^{d}$. It can be seen that $G$ is globally rigid in $\mathbb{R}$ if and only if $G$ is equal to $K_{2}$ or is 2-connected. Globally rigid graphs in $\mathbb{R}^{2}$ are characterised by a combination of results due to Hendrickson [14], Connelly [6], and Jackson and Jordán [16]. No characterisations are known in $\mathbb{R}^{d}$ when $d \geq 3$.

More generally we can consider the number of realisations which are equivalent to, i.e. have the same edge lengths as, a given generic realisation of a rigid graph ${ }^{1}$ in $\mathbb{R}^{d}$. It is known that this number is finite, see $[4,27]$, but in contrast to the case when the realisation is unique, the number of equivalent realisations may depend on both the graph and the realisation when $d \geq 2$, see Figures 1 and 2. Bounds on the maximum number of equivalent realisations, where the maximum is taken over all possible realisations of a given graph, are obtained by Borcea and Streinu in [4], and this number is determined exactly for generic realisations of graphs with a connected rigidity matroid in $\mathbb{R}^{2}$ by Jackson, Jordán, and Szabadka in [17]. Results and algorithms for calculating all equivalent realisations of frameworks which arise as backbones of protein molecules in $\mathbb{R}^{3}$ are given by Liberti et al. in [21, 22, 23].

The set of all realisations which are equivalent to a given realisation can be represented as the set of solutions to a system of quadratic equations. In this setting it is natural to consider the number of complex solutions. This number gives an upper bound on the number of real solutions which often plays a crucial role in calculating the exact number of real solutions, see for example $[7,9,33]$. In addition, the number of complex solutions is much better behaved than the number of real solutions. For example, we shall show that the number of complex solutions is the same for all generic

\footnotetext{
${ }^{1}$ Intuitively, a graph is rigid in $\mathbb{R}^{d}$ if every generic realisation in $\mathbb{R}^{d}$ is 'locally unique'. A formal definition of rigidity will be given in the next section.
} 


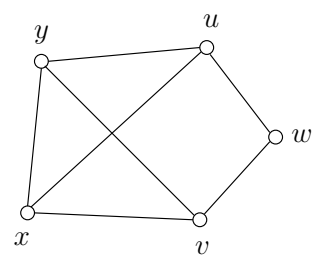

Figure 1: A realisation of a graph $G$ in $\mathbb{R}^{2}$. The only other equivalent realisation is obtained by reflecting the vertex $w$ in the line through $\{u, v\}$.
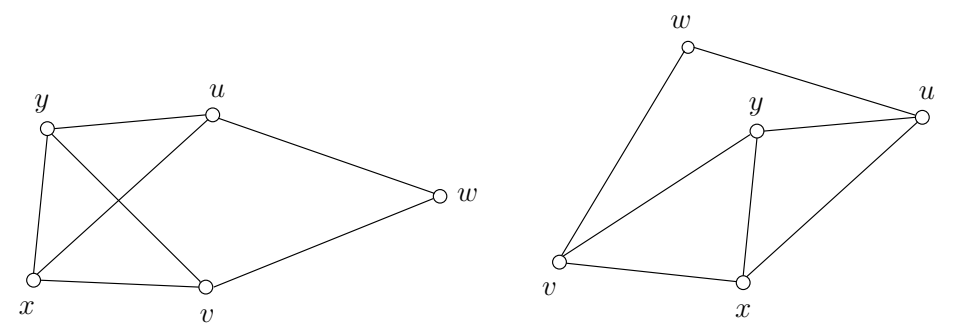

Figure 2: Two equivalent realisations of the graph $G$ of Figure 1 in $\mathbb{R}^{2}$. Two other equivalent realisation can be obtained from these by reflecting the vertex $w$ in the line through $\{u, v\}$, giving four different equivalent realisations in $\mathbb{R}^{2}$. 
realisations of a given rigid graph. The realisations of the graph $G$ shown in Figures 1 and 2 both have four equivalent complex realisations. Only two of these are real for the realisations shown in Figure 1, but all four are real in Figure 2.

Gortler and Thurston [13] recently showed that a graph has a unique generic realisation in $\mathbb{C}^{d}$ if and only if it has a unique generic realisation in $\mathbb{R}^{d}$. This implies that the above mentioned characterisations of globally rigid graphs in $\mathbb{R}^{d}$ for $d=1,2$ extend immediately to $\mathbb{C}^{d}$, and explains the apparent inconsistency that having a unique real realisation is a generic property whereas the number of different real realisations is not.

We will concentrate on the 2-dimensional case in this paper. We review notation and terminology for geometric rigidity theory in Section 2. We give some preliminary results from algebraic geometry in Section 3 and use them to show that the number, $c(G)$, of complex realisations of a rigid graph $G$ which are equivalent to a given generic realisation is the same for all generic realisations. We also show that, if $G$ is isostatic i.e. $G$ is rigid but $G-e$ is not rigid for all edges $e$ of $G$, then $c(G)$ is bounded below by the number of rigid realisations of $G$ which are equivalent to any given realisation.

We consider the effect of graph operations on $c(G)$ in Section 4 . It is known that a type 1 Henneberg move doubles $c(G)$. We will show that a type 2 Henneberg move on a redundant edge does not increase $c(G)$. We also show that the vertex splitting move increases $c(G)$ by a factor of at least two when $G$ is isostatic and that the same result holds for the maximum number of pairwise equivalent generic real realisations of $G$. We use this to deduce that every planar isostatic graph on $n$ vertices has at least $2^{n-3}$ pairwise equivalent generic real realisations. We use our result for Henneberg type 2 moves to give a short proof that the characterization of graphs with unique generic realisations in $\mathbb{R}^{2}$ extends to $\mathbb{C}^{2}$ in Section 5 .

In Section 6, we consider operations which glue two graphs $G_{1}, G_{2}$ together by either associating two pairs of vertices in each graph or by adding three edges between them, and show how $c(G)$ can be computed from $c\left(G_{1}\right)$ and $c\left(G_{2}\right)$. We use our results to determine $c(G)$ for a family of quadratically solvable graphs and for graphs with a connected rigidity matroid, and show that the graphs in both families have $c(G)$ pairwise equivalent generic real realisations in Section 7 . We close with a short section of examples and open problems. 


\section{Definitions and notation}

A complex (real) realisation of a graph $G=(V, E)$ is a map $p$ from $V$ to $\mathbb{C}^{2}$ $\left(\mathbb{R}^{2}\right)$. We also refer to the ordered pair $(G, p)$ as a framework. A complex, or real, framework $(G, p)$ is generic if the set of all coordinates of the points $p(v), v \in V$, is algebraically independent over $\mathbb{Q}$.

For $P=(x, y) \in \mathbb{C}^{2}$ let $d(P)=x^{2}+y^{2}$ and $\|P\|=\left(|x|^{2}+|y|^{2}\right)^{1 / 2}$, where |.| denotes the modulus of a complex number. Two frameworks $(G, p)$ and $(G, q)$ are equivalent if $d(p(u)-p(v))=d(q(u)-q(v))$ for all $u v \in E$, and are congruent if $d(p(u)-p(v))=d(q(u)-q(v))$ for all $u, v \in V$.

A framework $(G, p)$ is complex, respectively real, rigid if there exists an $\epsilon>0$ such that every complex, respectively real, framework $(G, q)$ which is equivalent to $(G, p)$ and satisfies $\|(p(v)-q(v) \|<\epsilon$ for all $v \in V$, is congruent to $(G, p)$. Equivalently, every continuous motion of the points $p(v), v \in V$, in $\mathbb{C}^{2}$, respectively $\mathbb{R}^{2}$, which respects the length constraints results in a framework which is congruent to $(G, p)$. Note that real rigidity considers only the real frameworks which are equivalent to a given real framework, whereas complex rigidity considers all equivalent complex frameworks, some or all of which may in fact be real.

The rigidity matrix of a framework $(G, p)$ is the matrix $R(G, p)$ of size $|E| \times 2|V|$, where, for each edge $v_{i} v_{j} \in E$, in the row corresponding to $v_{i} v_{j}$, the entries in the two columns corresponding to vertices $v_{i}$ and $v_{j}$ contain the two coordinates of $\left(p\left(v_{i}\right)-p\left(v_{j}\right)\right)$ and $\left(p\left(v_{j}\right)-p\left(v_{i}\right)\right)$, respectively, and the remaining entries are zeros. The framework is infinitessimally rigid if $\operatorname{rank} R(G, p)=2|V|-3$. (We always have $\operatorname{rank} R(G, p) \leq 2|V|-3$ since its null space always contains three linearly independent vectors corresponding to two translations and a rotation of the framework.)

Asimow and Roth [1] showed that infinitesimal rigidity is a sufficient condition for the real rigidity of $(G, p)$, and that the two properties are equivalent when $(G, p)$ is generic. This implies that real rigidity is a generic property and we say that $G$ is rigid if some/every generic real realisation of $G$ is real rigid. Theorem 3.6 below implies that complex rigidity is also a generic property and that a graph $G$ is complex rigid if and only if it is real rigid. This allows us to describe a graph as being rigid without the need to distinguish between real and complex rigidity. We say that $G$ is isostatic if it is minimally rigid i.e. $G$ is rigid but $G-e$ is not rigid for all edges $e$ of $G$. Isostatic graphs are characterised by a result of Laman [20] and this characterization was extended to all rigid graphs by Lovász and Yemini [24]. We refer the 
reader to [35] for more information on the rigidity of graphs.

Given a complex or real framework $(G, p)$, the fact that an algebraic variety can only contain finitely many isolated points implies that the maximum number of pairwise non-congruent rigid frameworks which are equivalent to $(G, p)$ is finite. We denote the number of such complex, or real, frameworks by $c(G, p)$, and $r(G, p)$, respectively. We will mostly be concerned with the case when $G$ is rigid and $(G, p)$ is generic. In this case all equivalent frameworks are rigid and hence $c(G, p)$, and $r(G, p)$, will count the total number of complex, respectively real, frameworks which are non-congruent and equivalent to $(G, p)$.

\section{Preliminary results}

In this section we set up the machinery which we will use to count the number of rigid frameworks which are equivalent to a given framework. We first show that for most frameworks $(G, p)$, we can choose a canonical representative in each congruence class of the set of all equivalent frameworks. We then state some standard results from algebraic geometry which will help us count the number of such canonical representatives. Finally, we apply our results to generic frameworks and show, in particular, that the number of canonical representatives is the same whenever $(G, p)$ is generic and rigid.

\subsection{Canonical position}

We say that a framework $(G, p)$ with $G=(V, E), V=\left\{v_{1}, v_{2}, \ldots, v_{n}\right\}$ and $n \geq 3$ is in canonical position (with respect to $\left.v_{1}, v_{2}, v_{3}\right)$ if $p\left(v_{1}\right)=(0,0)$, $p\left(v_{2}\right)=\left(0, b_{2}\right)$ with $b_{2} \neq 0$ and $\operatorname{Arg} b_{2} \in(0, \pi]$, and $p\left(v_{3}\right)=\left(a_{3}, b_{3}\right)$ with either $a_{3}=0$ or $\operatorname{Arg} a_{3} \in(0, \pi]$. It is collinear if $p(u)-p(v) \in\langle s\rangle$ for all $u, v \in V$, for some fixed $s \in \mathbb{C}^{2}$, respectively $s \in \mathbb{R}^{2}$.

Our first result tells us that most complex frameworks are congruent to a framework in canonical position. Its proof is given in the Appendix.

Lemma 3.1 Let $(G, p)$ be a complex framework, $v_{1}, v_{2}, v_{3}$ be vertices of $G$, and $S$ be the set of all equivalent frameworks. Suppose that $d\left(p\left(v_{1}\right)-p\left(v_{2}\right)\right) \neq$ 0 . Then each congruence class in $S$ has a unique representative $(G, q)$ which is in canonical position with respect to $v_{1}, v_{2}, v_{3}$. Furthermore:

(a) if $(G, p)$ is not collinear, then each congruence class in $S$ has exactly four 
realisations $(G, q)$ with $q\left(v_{1}\right)=(0,0)$ and $q\left(v_{2}\right)=\left(0, b_{2}\right)$ for some $b_{2} \in \mathbb{C} \backslash\{0\}$, and exactly two of these realisations have $\operatorname{Arg} b_{2} \in(0, \pi]$;

(b) if $(G, p)$ is collinear, then each congruence class in $S$ has exactly two realisations $(G, q)$ with $q\left(v_{1}\right)=(0,0)$ and $q\left(v_{2}\right)=\left(0, b_{2}\right)$ for some $b_{2} \in \mathbb{C} \backslash\{0\}$.

The frameworks $(G, p)$ which cannot be placed in canonical position are those which have $d(p(u)-p(v))=0$ for all vertices $u, v$ of $G$ i.e. $p(u)-p(v) \in$ $\langle(1, i)\rangle \cup\langle(1,-i)\rangle$, for all $u, v \in V$.

\subsection{Results from Algebraic Geometry}

Given a subfield $K$ of $\mathbb{C}$ and a point $p \in \mathbb{C}^{n}$ we use $K(p)$ to denote the field extension of $K$ by the coordinates of $p$. We say that $p$ is generic over $K$ if its components form an algebraically independent set over $K$. Given fields $K \subseteq L \subseteq \mathbb{C}$ with $L$ a finitely generated field extension of $K$, the transcendence degree of $L$ over $K, \operatorname{td}[L: K]$, is the cardinality of a largest subset of $L$ which is algebraically independent over $K$, see [29, Section 18.1]. (It follows from the Steinitz exchange axiom, see [29, Lemma 18.4], that every set of elements of $L$ which is algebraically independent over $K$ can be extended to a set of $\operatorname{td}[L: K]$ elements which is algebraically independent over $K$.) We use $\bar{K}$ to denote the algebraic closure of $K$ in $\mathbb{C}$. Note that $\operatorname{td}[\bar{K}: K]=0$.

We use $K\left[X_{1}, X_{2}, \ldots, X_{n}\right]$ to denote the ring of polynomials in the indeterminates $X_{1}, X_{2}, \ldots, X_{n}$ with coefficients in $K$ and $K\left(X_{1}, X_{2}, \ldots, X_{n}\right)$ to denote its field of fractions. Given a multivariate polynomial function $f: \mathbb{C}^{n} \rightarrow \mathbb{C}^{m}$ we use $\left.d f\right|_{x}$ to denote the Jacobean matrix of $f$ evaluated at a point $x \in \mathbb{C}^{n}$. We will need two standard results on generic points in $\mathbb{C}^{n}$. Their proofs are given in the Appendix.

Lemma 3.2 Let $f: \mathbb{C}^{n} \rightarrow \mathbb{C}^{m}$ by $f(p)=\left(f_{1}(p), f_{2}(p), \ldots, f_{m}(p)\right)$, where $f_{i} \in \mathbb{Q}\left[X_{1}, X_{2}, \ldots, X_{n}\right]$ for $1 \leq i \leq m$. Let $W(p)=\left\{q \in \mathbb{C}^{n}: f(p)=f(q)\right\}$ for each $p \in \mathbb{C}^{n}$.

(a) If If $p$ is generic and rank $\left.d f\right|_{p}=m$ then $f(p)$ is generic.

(b) If $\operatorname{td}[\mathbb{Q}(f(p)): \mathbb{Q}]=n$ then $p$ is generic and $\overline{\mathbb{Q}(p)}=\overline{\mathbb{Q}(f(p))}$.

(c) If $p$ is generic and rank $\left.d f\right|_{p}=n$ then $\overline{\mathbb{Q}(p)}=\overline{\mathbb{Q}(f(p))}, W(p)$ is finite, and $|W(p)|=|W(q)|$ for all generic $q \in \mathbb{C}^{n}$.

Lemma 3.3 Let $X_{1}, X_{2}, \ldots, X_{n}$ and $D_{1}, D_{2}, \ldots, D_{t}$ be indeterminates and let $f_{i} \in K\left[X_{1}, X_{2}, \ldots, X_{n}, D_{1}, D_{2}, \ldots, D_{t}\right]$ for all $1 \leq i \leq m$, for some field 
$K$ with $\mathbb{Q} \subseteq K \subseteq \mathbb{C}$. For each $d \in \mathbb{C}^{t}$ let $V_{d}=\left\{x \in \mathbb{C}^{n}: f_{i}(x, d)=\right.$ 0 for all $1 \leq i \leq m\}$. Then $V_{d} \neq \emptyset$ for some $d \in \mathbb{C}^{t}$ with $\operatorname{td}[K(d): K]=t$ if and only if $V_{d} \neq \emptyset$ for all $d \in \mathbb{C}^{t}$ with $\operatorname{td}[K(d): K]=t$.

\subsection{Generic frameworks}

Let $(G, p)$ be a framework with $G=(V, E), V=\left\{v_{1}, v_{2}, \ldots, v_{n}\right\}$ and $E=$ $\left\{e_{1}, e_{2}, \ldots, e_{m}\right\}$. We view $p$ as a point $p=\left(p\left(v_{1}\right), p\left(v_{2}\right), \ldots, p\left(v_{n}\right)\right)$ in $\mathbb{C}^{2 n}$. The rigidity $\operatorname{map}_{G}: \mathbb{C}^{2 n} \rightarrow \mathbb{C}^{m}$ is given by $d_{G}(p)=\left(\ell\left(e_{1}\right), \ell\left(e_{2}\right), \ldots, \ell\left(e_{m}\right)\right)$, where $\ell\left(e_{i}\right)=d(p(u)-p(v))$ when $e_{i}=u v$. Note that the evaluation of the Jacobian of the rigidity map $d_{G}$ at the point $p \in \mathbb{C}^{2 n}$ is twice the rigidity matrix of $(G, p)$. When $H$ is a subgraph of $G$, we will simplify notation and write $d_{H}(p)$ rather that $d_{H}\left(\left.p\right|_{H}\right)$.

A framework $(G, p)$ is said to be quasi-generic if it is congruent to a generic framework. Lemma 3.2 implies the following result for quasi-generic frameworks. A detailed proof is given in the Appendix.

Lemma 3.4 Let $(G, p)$ be a quasi-generic complex framework with vertices $v_{1}, v_{2}, \ldots, v_{n}$. Then $d\left(p\left(v_{i}\right)-p\left(v_{j}\right)\right) \neq 0$ for all $1 \leq i<j \leq n$ and $\operatorname{td}\left[\mathbb{Q}\left(d_{G}(p)\right): \mathbb{Q}\right]=\operatorname{rank} R(G, p)$. Furthermore, if $G$ is rigid, $p\left(v_{1}\right)=(0,0)$ and $p\left(v_{2}\right)=\left(0, b_{2}\right)$, then $\overline{\mathbb{Q}(p)}=\overline{\mathbb{Q}\left(d_{G}(p)\right)}$.

We can use Lemma 3.4 to show that the set of quasi-generic realisations of a rigid graph is closed under equivalence.

Lemma 3.5 Suppose $(G, p)$ is a rigid generic framework on $n \geq 3$ vertices and $(G, q)$ is equivalent to $(G, p)$. Then $(G, q)$ is quasi-generic.

Proof. Lemma 3.4 and the hypothesis that $(G, p)$ is rigid imply that

$$
\operatorname{td}\left[\mathbb{Q}\left(d_{G}(p)\right): \mathbb{Q}\right]=\operatorname{rank} R(G, p)=2 n-3 .
$$

We can now use Lemma 3.1 and the fact that $d_{G}(q)=d_{G}(p)$ to deduce that $(G, q)$ is congruent to a framework $\left(G, q^{*}\right)$ in canonical position with respect to three vertices $v_{1}, v_{2}, v_{3}$ of $G$. Lemma 3.4 gives $\operatorname{td}\left[\mathbb{Q}\left(q^{*}\right): \mathbb{Q}\right]=$ $\operatorname{td}\left[\mathbb{Q}\left(d_{G}(p)\right): \mathbb{Q}\right]=2 n-3$ and hence the last $2 n-3$ coordinates of $q^{*}$ are algebraically independent. We can now apply a translation and rotation to $\left(G, q^{*}\right)$ to transform it to a generic framework $(G, \tilde{q})$. Since $(G, q)$ is congruent to $(G, \tilde{q})$, it is quasi-generic. 
Theorem 3.6 Suppose $(G, p)$ is a quasi-generic complex realisation of a rigid graph $G=(V, E)$. Let $S$ be the set of all equivalent realisations of $G$. Then the number of congruence classes in $S$ is finite. Furthermore, this number is the same for all generic realisations of $G$.

Proof. The theorem is trivially true when $|V| \leq 2$ so we may assume that $|V| \geq 3$. We may also assume, by Lemmas 3.1 and 3.4 , that $(G, p)$ is in canonical position and that each congruence class of $S$ contains exactly four frameworks $(G, q)$ in which the first three coordinates of $q$ are zero. For each of these four frameworks, the last $2 n-3$ coordinates will be algebraically independent by Lemma 3.4. The theorem now follows by applying Lemma $3.2(\mathrm{c})$ to the map $f_{G}: \mathbb{C}^{2 n-3} \rightarrow \mathbb{C}^{m}$ given by $f_{G}(x)=d_{G}(0,0,0, x)$ evaluated at the point $x=\hat{p}$, where $\hat{p}$ is the projection of $p$ onto its last $2 n-3$ coordinates.

As mentioned in the Introduction, we denote this common value of $c(G, p)$ over all generic realisations of $G$ by $c(G)$. We close this section by showing that we may obtain a lower bound on $c(G)$ when $G$ is isostatic by using a non-generic realisation of $G$. Our proof uses the concept of the multiplicity of an isolated solution of a system of polynomial equations. We refer the reader to [32, page 224] for a formal definition but note that an isolated solution $p \in \mathbb{C}^{n}$ of a system of $n$ equations in $n$ variables has multiplicity one if the Jacobean of the system has rank $n$ at $p$ and has multiplicity at least two if the Jacobean has rank less than $n$.

Let $S$ be the set of all rigid frameworks which are equivalent to a given framework $(G, p), \Omega(G, p)$ be the partition of $S$ into congruence classes and $\Omega^{\prime}(G, p)$ be the set of all congruence classes in $\Omega(G, p)$ which contain frameworks which are rigid but are not infinitesimally rigid and not collinear. By definition we have $c(G, p)=|\Omega|$. Let $c^{\prime}(G, p)=\left|\Omega^{\prime}\right|$.

Theorem 3.7 Suppose $(G, p)$ is a realisation of an isostatic graph $G=$ $(V, E)$ with $d\left(p\left(v_{1}\right)-p\left(v_{2}\right)\right) \neq 0$ for some $v_{1} v_{2} \in E$. Then

$$
c(G) \geq c(G, p)+c^{\prime}(G, p) .
$$

Proof. Let $(G, q)$ be a generic realisation of $G$. Since $G$ is isostatic and $(G, q)$ is generic, $d_{G}(q)$ is generic over $\mathbb{Q}$ by Lemma 3.4. Let $W_{p}$ and $W_{q}$ be the set of all $t \in \mathbb{C}^{2|V|}$ such that $t\left(v_{1}\right)=(0,0)$, the first component of $t\left(v_{2}\right)$ is zero, and $(G, t)$ is equivalent to $(G, p)$, respectively $(G, q)$. Then Lemmas 3.1 
and 3.4 imply that $W_{q}$ is a complex algebraic variety defined for a generic set of parameters $d_{G}(q)$ and has exactly $4 c(G)$ points. Let $m_{i}(q)$ be the number of isolated points of $W_{q}$ with multiplicity $i$. Then $m_{i}(q)=0$ for $i \geq 2$ because every framework equivalent to $(G, q)$ is infinitesimally rigid by Lemma 3.4. Hence $\Sigma_{i \geq 1} i m_{i}(q)=4 c(G)$.

Similarly let $m_{i}(p)$ be the number of isolated points of $W_{p}$ with multiplicity $i$. Since $W_{q}$ is defined by a set of $|E|$ polynomials in $2|V|-3$ variables with $|E|=2|V|-3$ and $d_{G}(p)$ is a specialisation of $d_{G}(q)$, we have $\Sigma_{i \geq 1} i m_{i}(p) \leq \Sigma_{i \geq 1} i m_{i}(q)$ by [32, Theorem 7.1.6].

Let $(G, \tilde{p})$ be a rigid framework which is equivalent to $(G, p)$. Then Lemma 3.1 implies that $W_{p}$ contains at least two isolated points which are congruent to $\tilde{p}$ if $(G, p)$ is collinear and at least four isolated points which are congruent to $\tilde{p}$ if $(G, p)$ is not collinear. Furthermore, each isolated point of $W_{p}$ corresponding to a rigid framework which is not infinitesimally rigid (and in particular each isolated point corresponding to a rigid collinear framework) has multiplicity at least two, and each isolated point corresponding to an infinitesimally rigid framework has multiplicity one. Hence

$$
4 c(G, p)+4 c^{\prime}(G, p) \leq \sum_{i \geq 1} i m_{i}(p) \leq \sum_{i \geq 1} i m_{i}(q)=4 c(G)
$$

Note that Theorem 3.7 also holds when $d\left(p\left(v_{1}\right)-p\left(v_{2}\right)\right)=0$ for all $v_{1} v_{2} \in$ $E$ since in this case we have $c(G, p)=1, c^{\prime}(G, p)=0$ and $c(G) \geq 1$.

The result [32, Theorem 7.1.6] we used in the proof of Theorem 3.7 is obtained using homotopic continuation. A purely algebraic proof for the case when all frameworks equivalent to $(G, p)$ are rigid can be obtained using [15, Chapter XI].

It is not difficult to construct frameworks which show that strict inequality can hold in Theorem 3.7. For example label the vertices of $K_{4}$ as $v_{1}, v_{2}, v_{3}, v_{4}$, let $H=K_{4}-v_{3} v_{4}$ and let $G$ be obtained by adding a new vertex $v_{5}$ and two new edges $v_{5} v_{3}, v_{5} v_{4}$ to $H$. It is straightforward to show that $c(G)=$ 4 , for example by using Lemma 4.1 below. However the realisation $(G, p)$ given by $p\left(v_{1}\right)=(0,0), p\left(v_{2}\right)=(0,1), p\left(v_{3}\right)=(1,1), p\left(v_{4}\right)=(-1,1)$, and $p\left(v_{5}\right)=(2,3)$ has $c(G, p)=2$. This follows because every realisation $(H, q)$ which is equivalent but not congruent to $\left(H,\left.p\right|_{H}\right)$ has $q\left(v_{3}\right)=q\left(v_{4}\right)$ and hence cannot be extended to a realisation of $G$ which is equivalent to $(G, p)$ 
(because $\left.d\left(p\left(v_{5}\right)-p\left(v_{3}\right)\right) \neq d\left(p\left(v_{5}\right)-p\left(v_{4}\right)\right)\right)$. Thus all realisations equivalent to $(G, p)$ are extensions of $\left(H,\left.p\right|_{H}\right)$ and there are exactly two ways to do this.

Note also that the conclusion of Theorem 3.7 does not hold for rigid graphs which are not isostatic. For example, label the vertices of $K_{5}$ as $v_{1}, v_{2}, v_{3}, v_{4}, v_{5}$, and let $G=K_{5}-v_{4} v_{5}$. Then $c(G)=1$ because $G$ is globally rigid. On the other hand, any rigid realisation $(G, p)$ with $p\left(v_{1}\right), p\left(v_{2}\right)$ and $p\left(v_{3}\right)$ collinear has $c(G, p) \geq 2$ since we may obtain an equivalent but noncongruent realisation by reflecting $p\left(v_{4}\right)$ in the line joining $p\left(v_{1}\right), p\left(v_{2}\right)$ and $p\left(v_{3}\right)$.

\section{Graph construction moves}

We first consider the effect of Henneberg moves on the number of equivalent complex realisations of a rigid graph. The type 1 Henneberg move on a graph $H$ adds a new vertex $v$ and two new edges $v x, v y$ from $v$ to distinct vertices $x, y$ of $H$. The type 2 Henneberg move deletes an edge $x y$ from $H$ and adds a new vertex $v$ and three new edges $v x, v y, v z$ from $v$ to $x, y$ and another vertex $z$ of $H$ distinct from $x, y$.

It is straightforward to show that applying the type 1 move will double the number of realisations, see for example $[4,33]$.

Lemma 4.1 Let $G=(V, E)$ be a rigid graph with at least four vertices, $v_{n} \in V$ with $N\left(v_{n}\right)=\left\{v_{1}, v_{2}\right\}$, and $H=G-v_{n}$. Then $c(G)=2 c(H)$.

We next consider type 2 moves. We need the following result which is an extension of [17, Lemma 4.1] to complex frameworks. Its proof uses ideas from simplified versions of the proof of [17, Lemma 4.1] given in $[25,31]$.

Lemma 4.2 Let $(G, p)$ be a quasi-generic complex framework and $v_{n} \in V$ with $N\left(v_{n}\right)=\left\{v_{1}, v_{2}, v_{3}\right\}$. Suppose that $(G, q)$ is a complex realisation of $G$ which is equivalent to $(G, p)$. If $G-v_{n}$ is rigid then $d\left(p\left(v_{i}\right)-p\left(v_{j}\right)\right)=$ $d\left(q\left(v_{i}\right)-q\left(v_{j}\right)\right)$ for all $1 \leq i<j \leq 3$.

Proof. By symmetry we need only show that $d\left(p\left(v_{1}\right)-p\left(v_{2}\right)\right)=d\left(q\left(v_{1}\right)-\right.$ $\left.q\left(v_{2}\right)\right)$. Label the vertices of $G$ as $v_{1}, \ldots, v_{n}$ and put $p\left(v_{i}\right)=p_{i}=\left(p_{i, 1}, p_{i, 2}\right)$ and $q\left(v_{i}\right)=q_{i}=\left(q_{i, 1}, q_{i, 2}\right)$ for all $1 \leq i \leq n$. Since $G-v_{n}$ is rigid and 
$d\left(v_{n}\right)=3, G$ is rigid. By applying Lemma 3.1 to both $(G, p)$ and $(G, q)$, we may suppose that $p_{1,1}=p_{1,2}=p_{2,2}=0$ and $q_{1,1}=q_{1,2}=q_{2,2}=0$. ${ }^{2}$ Then

$$
d\left(p_{1}-p_{2}\right)-d\left(q_{1}-q_{2}\right)=p_{2,1}^{2}-q_{2,1}^{2}
$$

so it will suffice to show that $p_{2,1}^{2}-q_{2,1}^{2}=0$.

Let $p^{\prime}=\left.p\right|_{G-v_{n}}, q^{\prime}=\left.q\right|_{G-v_{n}}, K=\mathbb{Q}\left(p^{\prime}\right)$ and $L=\mathbb{Q}\left(q^{\prime}\right)$. Consider the equivalent frameworks $\left(G-v_{n}, p^{\prime}\right)$ and $\left(G-v_{n}, q^{\prime}\right)$. Applying Lemma 3.4 to $G-v_{n}$, we have $\bar{K}=\bar{L}$. Thus $q_{2,1}, q_{3,1}, q_{3,2} \in \bar{K}$. Since $(G, q)$ is equivalent to $(G, p)$, we have the following equations.

$$
\begin{aligned}
q_{n, 1}^{2}+q_{n, 2}^{2} & =p_{n, 1}^{2}+p_{n, 2}^{2} \\
\left(q_{n, 1}-q_{2,1}\right)^{2}+q_{n, 2}^{2} & =\left(p_{n, 1}-p_{2,1}\right)^{2}+p_{n, 2}^{2} \\
\left(q_{n, 1}-q_{3,1}\right)^{2}+\left(q_{n, 2}-q_{3,2}\right)^{2} & =\left(p_{n, 1}-p_{3,1}\right)^{2}+\left(p_{n, 2}-p_{3,2}\right)^{2}
\end{aligned}
$$

Subtracting (1) from (2) and (3) we obtain

$$
\begin{aligned}
q_{n, 1} & =\frac{p_{2,1}}{q_{2,1}} p_{n, 1}+\frac{q_{2,1}^{2}-p_{2,1}^{2}}{2 q_{2,1}} \\
q_{n, 2} & =\frac{p_{3,1}}{q_{3,2}} p_{n, 1}+\frac{p_{3,2}}{q_{3,2}} p_{n, 2}-\frac{q_{3,1}}{q_{3,2}} q_{n, 1}+\frac{q_{3,1}^{2}-p_{3,1}^{2}+q_{3,2}^{2}-p_{3,2}^{2}}{2 q_{3,2}}
\end{aligned}
$$

We may use (4) to eliminate $q_{n, 1}$ from the right hand side of (5) to obtain a matrix equation for $q_{n}$ of the form

$$
q_{n}=A p_{n}+b
$$

where $A$ is a $2 \times 2$ lower triangular matrix with entries in $\bar{K}$ and $b \in \bar{K}^{2}$. Rewriting (1) as $q_{n}^{T} q_{n}=p_{n}^{T} p_{n}$ and then substituting for $q_{n}$ using (6) we obtain

$$
p_{n}^{T}\left(A^{T} A-I\right) p_{n}+2 b^{T} A p_{n}+b^{T} b=0 .
$$

This is a polynomial equation for the components of $p_{n}$ with coefficients in $\bar{K}$. Since $\operatorname{td}[\mathbb{Q}(p): \mathbb{Q}]=2 n-3$ by Lemma $3.4,\left\{p_{n, 1}, p_{n, 2}\right\}$ is algebraically independent over $\bar{K}$. This implies that the polynomial on the left hand side of (7) is identically zero. In particular $A^{T} A=I$ and, since $A$ is lower triangular, $A$ must be a diagonal matrix with \pm 1 entries on the diagonal. In particular $a_{1,1}=p_{2,1} / q_{2,1}= \pm 1$ and hence $p_{2,1}^{2}-q_{2,1}^{2}=0$.

\footnotetext{
${ }^{2}$ We have switched the order of the coordinate axes from that given in Lemma 3.1 since it makes the remainder of the proof more straightforward.
} 
Lemma 4.3 Let $G=(V, E)$ be a rigid graph, $v_{n} \in V$ with $N\left(v_{n}\right)=\left\{v_{1}, v_{2}, v_{3}\right\}$, and let $H$ be obtained from $G-v_{n}$ by adding the edges $e_{1}=v_{1} v_{2}, e_{2}=v_{2} v_{3}$ and $e_{3}=v_{1} v_{3}$ whenever they do not already exist in $G-v_{n}$. Suppose that $G-v_{n}$ is rigid. Then $c(G)=c(H)$.

Proof. Let $(G, p)$ be a generic realisation of $G$ and $\left(G, p^{\prime}\right)$ be a realisation which is congruent to $(G, p)$ and in canonical position. Let $S$ be the set of all realisations $(G, q)$ which are equivalent to $(G, p)$ and in canonical position. Similarly, let $S^{*}$ be the set of all realisations $\left(H, q^{*}\right)$ which are equivalent to $\left(H,\left.p\right|_{H}\right)$ and in canonical position. By Lemma 3.1, $|S|=c(G)$ and $\left|S^{*}\right|=$ $c(H)$.

Let $F$ be a complete graph with vertex set $\left\{v_{1}, v_{2}, v_{3}, v_{n}\right\}$. Then Lemma 4.2 implies that $\left(F,\left.q\right|_{V(F)}\right)$ is congruent to $\left(F,\left.p^{\prime}\right|_{V(F)}\right)$ for all $(G, q) \in S$. Lemma 3.1 now gives $q\left(v_{i}\right)=p^{\prime}\left(v_{i}\right)$ for all $i \in\{1,2,3, n\}$ and all $(G, q) \in$ $S$. We may use a similar argument to deduce that $q^{*}\left(v_{i}\right)=p^{\prime}\left(v_{i}\right)$ for all $i \in\{1,2,3\}$ and all $\left(H, q^{*}\right) \in S^{*}$. This implies that the map $\theta: S \rightarrow S^{*}$ defined by $\theta(G, q)=\left(H,\left.q\right|_{V-v_{n}}\right)$ for all $(G, q) \in S$ is a bijection. Hence $c(G)=|S|=\left|S^{*}\right|=c(H)$.

Corollary 4.4 Let $G=(V, E)$ be a rigid graph, $v_{n} \in V$ with $N\left(v_{n}\right)=$ $\left\{v_{1}, v_{2}, v_{3}\right\}$, and $H=\left(G-v_{n}\right)+e_{1}$ where $e_{1}=v_{1} v_{2}$. Suppose that $G-v_{n}$ is rigid. Then $c(G) \leq c(H)$.

Proof. By Lemma 4.3, $c(G)=c\left(H \cup\left\{e_{2}, e_{3}\right\}\right) \leq c(H)$, where $e_{2}=v_{2} v_{3}$ and $e_{3}=v_{1} v_{3}$.

An edge $e$ in a rigid graph $G$ is redundant if $G-e$ is rigid. Corollary 4.4 tells us that if we extend a rigid graph $H$ by performing a Henneberg type 2 move on a redundant edge of $H$ then we do not increase $c(H)$. On the other hand it is not difficult to construct examples with $c(G)=1$ and $c(H)$ arbitrarily large.

It is an open problem to determine the effect that performing a Henneberg type 2 move on a non-redundant edge has on $c(H)$.

Problem 4.5 Do there exist universal constants $k_{1}, k_{2}>0$ such that if $H$ is a rigid graph and $G$ is obtained by performing a Henneberg type 2 move on a non-redundant edge of $H$, then $k_{1} c(H) \leq c(G) \leq k_{2} c(H)$ ? 
In particular, we do not know whether performing a Henneberg type 2 move on a non-redundant edge can decrease the number of complex realisations.

We next consider the operation of vertex splitting introduced by Whiteley in [34]. Given a vertex $v$ in a graph $H$, this move constructs a new graph $G$ from $H-v$ by partitioning the neighbours of $v$ into two sets $N_{1}, N_{2}$, adding two new vertices $v_{1}, v_{2}$ joined to $N_{1}, N_{2}$ respectively, then adding the edge $v_{1} v_{2}$ and another edge $v_{2} x$ for any $x \in N_{1}$. Whiteley showed that this move preserves rigidity. Since it also preserves the edge count, $G$ will be isostatic whenever $H$ is isostatic.

Lemma 4.6 Let $H$ be an isostatic graph on at least three vertices and $G$ be obtained from $H$ by applying the vertex splitting move. Then $c(G) \geq 2 c(H)$.

Proof. Suppose that $G$ is obtained from $H$ by splitting $v$ into $v_{1}, v_{2}$. Let $(H, p)$ be a generic realisation of $H$. Construct a realisation $(G, q)$ of $G$ by putting $q\left(v_{1}\right)=q\left(v_{2}\right)=p(v)$ and $q(u)=p(u)$ for all other vertices $u$. We will obtain a bound on $c(G)$ by applying Lemma 3.7 to $(G, q)$. It is straightforward to show that $c(G, q)=c(H, p)$. In addition, no framework which is equivalent to $(G, q)$ is infinitesimally rigid (because the row indexed by $v_{1} v_{2}$ in its rigidity matrix is zero) or collinear (because $(H, p)$ is generic). Hence $c^{\prime}(G, q)=c(G, q)$. Theorem 3.7 now gives

$$
c(G) \geq 2 c(G, q)=2 c(H, p)=2 c(H) .
$$

We can also obtain a lower bound on the number of real generic realisations produced by the vertex split operation. We need the following lemma.

Lemma 4.7 Suppose that $G=(V, E)$ is isostatic and $\left(G, s_{i}\right), 1 \leq i \leq m$, are distinct pairwise equivalent infinitesimally rigid real frameworks in canonical position with respect to three given vertices $v_{1}, v_{2}, v_{3}$. Then, for each $\epsilon>0$, there exist distinct quasi-generic pairwise equivalent real frameworks $\left(G, t_{i}\right)$, $1 \leq i \leq m$, in canonical position with respect to $v_{1}, v_{2}, v_{3}$, which satisfy $\left\|t_{i}-s_{i}\right\|<\epsilon$ for all $1 \leq i \leq m$.

Proof. Let $V=\left\{v_{1}, v_{2}, \ldots, v_{n}\right\}$ and $E=\left\{e_{1}, e_{2}, \ldots, e_{2 n-3}\right\}$. We associate each vector $\hat{p}=\left(b_{2}, a_{3}, b_{3}, \ldots, a_{n}, b_{n}\right) \in \mathbb{R}^{2 n-3}$ with a real framework $(G, p)$ where $p\left(v_{1}\right)=(0,0), p\left(v_{2}\right)=\left(0, b_{2}\right)$ and $p\left(v_{i}\right)=\left(a_{i}, b_{i}\right)$ for $i \geq 3$. We can 
now define a differentiable map $f_{G}: \mathbb{R}^{2 n-3} \rightarrow \mathbb{R}^{2 n-3}$ by taking $f_{G}(\hat{p})$ to be the ordered vector of squared edge lengths in the framework $(G, p)$. The rank of the Jacobean matrix $\left.d f_{G}\right|_{\hat{p}}$ is equal to the rank of the rigidity matrix of $(G, p)$ and hence rank $\left.d f_{G}\right|_{\hat{s}_{i}}=2 n-3$ for all $1 \leq i \leq m$. The inverse function theorem now implies that we can choose open neighbourhoods $N_{i}$ of $\hat{s}_{i}$ and $N$ of $f_{G}\left(\hat{s}_{i}\right)$ in $\mathbb{R}^{2 n-3}$ such that $f_{G}$ maps $N_{i}$ diffeomorphically onto $N$ for all $1 \leq i \leq m$. This allows us to choose a generic point $d \in N$ and points $\hat{t}_{i} \in N_{i}$ such that $\left\|t_{i}-s_{i}\right\|<\epsilon$ and $f_{G}\left(\hat{t}_{i}\right)=d$ for all $1 \leq i \leq m$. Since $d$ is generic and $\mathbb{Q}(d) \subseteq \mathbb{Q}\left(\hat{t}_{i}\right)$, the coordinates of $\hat{t}_{i}$ are algebraically independent and hence each framework $\left(G, t_{i}\right)$ is quasi-generic. We can ensure that $\left(G, t_{i}\right)$ is in canonical position with respect to $v_{1}, v_{2}, v_{3}$ by choosing $d$ such that $t_{i}$ is sufficiently close to $s_{i}$.

Theorem 4.8 Let $H$ be an isostatic graph on at least three vertices and let $G$ be obtained from $H$ by applying the vertex splitting move. Let $(H, p)$ be a generic real realisation of $H$. Then there exists a generic realisation $(G, t)$ of $G$ such that $r(G, t) \geq 2 r(H, p)$.

Proof. Suppose the vertex splitting move splits $v$ into $v_{1}, v_{2}$ and adds edges $v_{1} v_{2}$ and $v_{1} x$. We may suppose that $(H, p)$ is a quasi-generic real realisation of $H$ in canonical position with respect to $v, x, y$ for some vertex $y$ of $H$. We have $p(v)=(0,0)$ and $p(x)=(0, a)$ with $a$ generic. Note that all frameworks equivalent to $(H, p)$ are quasi-generic and hence infinitesimally rigid by Lemma 3.5. Construct a real realisation $(G, q)$ of $G$ by putting $q\left(v_{1}\right)=q\left(v_{2}\right)=p(v)$ and $\left.q\right|_{H}=p$, and let $S$ be the set of all equivalent real realisations $\left(G, q_{i}\right)$, in canonical position with respect to $v_{1}, x, y$. It is straightforward to show that $r(G, q)=|S|=r(H, p)$.

Let $\hat{G}$ be the graph obtained from $G$ by performing a Henneberg type 2 move which deletes the edge $v_{1} v_{2}$ and adds a new vertex $w$ and new edges $w v_{1}, w v_{2}, w x$. Let $(\hat{G}, \hat{q})$ be the framework obtained by putting $\hat{q}(w)=(a, 0)$ and $\left.\hat{q}\right|_{G}=q$, and let $\hat{S}$ be the set of all equivalent realisations $\left(\hat{G}, \hat{q}_{i}\right)$ which are in canonical position with respect to $v_{1}, x, y$ and satisfy $\hat{q}_{i}\left(v_{1}\right)=\hat{q}_{i}\left(v_{2}\right)=$ $(0,0)$. Then $\left(\hat{G}, \hat{q}_{i}\right) \in \hat{S}$ if and only if $\left(G,\left.\hat{q}_{i}\right|_{G}\right) \in S$ and $\hat{q}_{i}(w)=( \pm a, 0)$, so $|\hat{S}|=2|S|=2 r(H, p)$. In addition each $\left(\hat{G}, \hat{q}_{i}\right) \in \hat{S}$ is infinitesimally rigid. To see this suppose that $m$ is an infinitesimal motion of $\left(\hat{G}, \hat{q}_{i}\right)$ with $m\left(v_{1}\right)=m(x)=(0,0)$. Then $m(w)=(0,0)$ and so $m\left(v_{2}\right)=(0,0)$. It follows that $m$ induces an infinitesimal motion of the framework $\left(H, p_{i}\right)$ given by 
$p_{i}(v)=(0,0)$ and $\left.p_{i}\right|_{H-v}=\left.\hat{q}_{i}\right|_{H-v}$, which is zero on $v$ and $x$. The facts that $\left(H, p_{i}\right)$ is equivalent to $(H, p)$ and that all frameworks equivalent to $(H, p)$ are infinitesimally rigid now tells us that $m$ is identically zero.

We can now use Lemma 4.7 to deduce that, for all $\epsilon>0$, there exists a set $\hat{T}$ of infinitesimally rigid, pairwise equivalent, quasi-generic real frameworks with $|\hat{T}|=|\hat{S}|$ and such that each $\left(\hat{G}, \hat{t}_{i}\right) \in \hat{T}$ is in canonical position with respect to $v_{1}, x, y$, and satisfies $\left\|\hat{t}_{i}-\hat{q}_{i}\right\|<\epsilon$ for all $1 \leq i \leq|\hat{T}|$.

Let $T$ be the set of all frameworks $\left(G, t_{i}\right)$ where $t_{i}=\left.\hat{t}_{i}\right|_{G}$ and $\left(\hat{G}, \hat{t}_{i}\right) \in \hat{T}$. We will show that, for sufficiently small $\epsilon$, we have $|T|=|\hat{T}|$ and each $\left(G, t_{i}\right) \in T$ is infinitesimally rigid and equivalent to $\left(G, t_{1}\right)$. Recall that $\hat{q}_{i}\left(v_{2}\right)=(0,0)$ and $\hat{q}_{i}(w)=( \pm a, 0)$ for all $1 \leq i \leq|\hat{S}|$. Let $\hat{t}_{1}\left(v_{2}\right)=\left(a_{2}, b_{2}\right)$ and $\hat{t}_{1}(w)=\left(a^{\prime}, b^{\prime}\right)$. Then, for sufficiently small $\epsilon$, the fact that $\left\{v_{1}, x, w\right\}$ induce a triangle in $\hat{G}$ implies that $\hat{t}_{i}(w)=\left( \pm a^{\prime}, b^{\prime}\right)$. The fact that $\left\{v_{2}, x, w\right\}$ induces a triangle in $\hat{G}$ now implies that $\hat{t}_{i}\left(v_{2}\right)=\left(a_{2}, b_{2}\right)$ when $\hat{t}_{i}(w)=\left(a^{\prime}, b^{\prime}\right)$ and $\hat{t}_{i}\left(v_{2}\right)=\left(-a_{2}, b_{2}\right)$ when $\hat{t}_{i}(w)=\left(-a^{\prime}, b^{\prime}\right)$. This gives

$$
d\left(t_{i}\left(v_{2}\right)-t_{i}\left(v_{1}\right)\right)=a_{2}^{2}+b_{2}^{2}=d\left(t_{1}\left(v_{2}\right)-t_{1}\left(v_{1}\right)\right)
$$

so each $\left(G, t_{i}\right)$ is equivalent to $\left(G, t_{1}\right)$. The assertion that each $\left(G, t_{i}\right)$ is infinitesimally rigid now follows from the facts that $G$ is rigid and $\left(G, t_{i}\right)$ is quasi-generic.

It remains to show that $|T|=|\hat{T}|$. Choose $i, j$ with $1 \leq i<j \leq|\hat{T}|$. Since $\hat{q}_{i} \neq \hat{q}_{j}$, we have $\hat{q}_{i}(u) \neq \hat{q}_{j}(u)$ for some vertex $u$ of $\hat{G}$. If $u \neq w$ then the fact that $\hat{t}_{i}(u)$ and $\hat{t}_{j}(u)$ can be chosen to be arbitrarily close to $\hat{q}_{i}(u)$ and $\hat{q}_{j}(u)$, respectively, means we can ensure that $t_{i}(u)=\hat{t}_{i}(u) \neq \hat{t}_{j}(u)=t_{j}(u)$. Hence suppose that $u=w$. Interchanging $i, j$ if necessary, we have $\hat{q}_{i}(w)=(a, 0)$ and $q_{j}(w)=(-a, 0)$. This implies that $\hat{t}_{i}(w)=\left(a^{\prime}, b^{\prime}\right)$ and $\hat{t}_{j}(w)=\left(-a^{\prime}, b\right)$ and hence that $t_{i}\left(v_{2}\right)=\hat{t}_{i}\left(v_{2}\right)=\left(a_{2}, b_{2}\right)$ and $t_{j}\left(v_{2}\right)=\hat{t}_{j}\left(v_{2}\right)=\left(-a_{2}, b_{2}\right)$. Hence $t_{i} \neq t_{j}$ for all $1 \leq i<j \leq|\hat{T}|$.

We can now combine the above inequalities to deduce that

$$
r\left(G, t_{1}\right) \geq|T|=|\hat{T}|=|\hat{S}|=2|S|=2 r(H, p)
$$

and the result follows since $\left(G, t_{1}\right)$ is quasi-generic.

Theorem 4.9 Every planar isostatic graph $G=(V, E)$ has a generic realisation $(G, p)$ such that $r(G, p) \geq 2^{|V|-3}$. 
Proof. Every isostatic planar graph can be reduced to a 3-cycle by a sequence of edge contractions in such a way that each intermediate graph is planar and isostatic by $[10,27]$. Since each edge contraction reduces $|V|$ by one the result follows by induction using Theorem 4.8 and the fact that $K_{3}$ is globally rigid.

\section{Globally rigid graphs and globally linked pairs of vertices}

We first use Corollary 4.4 to characterise graphs $G$ with $c(G)=1$. Our characterization is the same as that given in [16] for globally rigid graphs in $\mathbb{R}^{2}$. (This result can be deduced immediately from the characterisation in [16] and the result of Gortler and Thurston mentioned in the Introduction that generic global rigidity in $\mathbb{R}^{d}$ and $\mathbb{C}^{d}$ are equivalent. We give our proof since it is short and direct.)

Theorem 5.1 Let $G=(V, E)$ be a graph with at least four vertices. Then $c(G)=1$ if and only if $G$ is 3-connected and redundantly rigid.

Proof. Necessity was proved for real (and hence also for complex) generic realisations in [14]. We prove sufficiency by induction on $|V|+|E|$. If $G$ has four vertices then $G=K_{4}$ and $c(G)=1$ since $G$ is complete. Hence suppose that $|V| \geq 5$. If $G-e$ is 3 -connected and redundantly rigid for some $e \in E$, then $c(G-e)=1$ by induction, and hence $c(G)=1$. Thus we may suppose that $G-e$ is not both 3 -connected and redundantly rigid. By [16, Theorem 6.1] there exists a vertex $v_{n} \in V$ with $N(v)=\left\{v_{1}, v_{2}, v_{3}\right\}$ such that $H=G-v_{n}+v_{1} v_{2}$ is 3 -connected and redundantly rigid. This implies in particular that $G-v_{n}$ is rigid. Induction and Corollary 4.4 now give $c(G) \leq c(H)=1$.

Let $(G, p)$ be a complex realisation of a rigid graph $G=(V, E)$ and $u, v \in V$. We say that $\{u, v\}$ is globally linked in $(G, p)$ if every equivalent complex realisation $(G, q)$ of $G$ has $d(p(u)-p(v))=d(q(u)-q(v))$. It can be seen that $u, v$ is globally linked in $(G, p)$ if and only if $c(G, p)=c(G+e, p)$, where $e=u v$. Theorem 3.6 now implies that the property of being globally linked is a generic property i.e. if $\{u, v\}$ is globally linked in some generic 
complex realisation of $G$ then $\{u, v\}$ is globally linked in all such realisations. We say that $\{u, v\}$ is globally linked in $G$ if $\{u, v\}$ is globally linked in some, or equivalently all, generic complex realisations of $G$.

The analogous concept for real realisations was introduced in [17]. (The situation for generic real realisations is more complicated as it is not necessarily true that if $\{u, v\}$ is globally linked in some generic real realisation of $G$ then $\{u, v\}$ is globally linked in all generic real realisations. For example the pair $u, v$ is globally linked in the real realisation in Figure 1, but not in Figure 2. This problem is circumvented in [17] by defining $\{u, v\}$ to be globally linked in $G$ in $\mathbb{R}^{2}$ if $\{u, v\}$ is globally linked in all generic real realisations of $G$.)

Our next result is analogous to a result for real realisations given in $[17$, Theorem 4.2].

Theorem 5.2 Let $(G, p)$ be a generic complex realisation of a graph $G=$ $(V, E)$ and $u, v, v_{1}, v_{2}, v_{3}, v_{n} \in V$ with $N\left(v_{n}\right)=\left\{v_{1}, v_{2}, v_{3}\right\}$ and $v_{n} \neq u, v$. Let $H=G-v_{n}+v_{1} v_{2}$. Suppose that $G-v_{n}$ is rigid and that $\{u, v\}$ is globally linked in $\left(H,\left.p\right|_{H}\right)$. Then $\{u, v\}$ is globally linked in $(G, p)$.

Proof. Suppose $(G, q)$ is equivalent to $(G, p)$. Let $p^{*}=\left.p\right|_{H}$ and $q^{*}=\left.q\right|_{H}$. Since $G-v_{n}=H-v_{1} v_{2}$ is rigid, Lemma 4.2 implies that $d\left(p\left(v_{1}\right)-p\left(v_{2}\right)\right)=$ $d\left(q\left(v_{1}\right)-q\left(v_{2}\right)\right)$. Hence $\left(H, p^{*}\right)$ and $\left(H, q^{*}\right)$ are equivalent. Since $\{u, v\}$ is globally linked in $\left(H, p^{*}\right)$, we have

$$
d(p(u)-p(v))=d\left(p^{*}(u)-p^{*}(v)\right)=d\left(q^{*}(u)-q^{*}(v)\right)=d(q(u)-q(v)) .
$$

Thus $\{u, v\}$ is globally linked in $(G, p)$.

The real analogue of Theorem 5.2 was used in [17, Section 5] to characterize when two vertices in a generic real realisation of an ' $\mathcal{M}$-connected graph' are globally linked in $\mathbb{R}^{2}$. We can show that the same characterization holds for complex realisations. We first need to introduce some new terminology.

A matroid $\mathcal{M}=(E, \mathcal{I})$, consists of a set $E$ together with a family $\mathcal{I}$ of subsets of $E$, called independent sets, which satisfy three simple axioms which capture the properties of linear independence in vector spaces, see [28]. Given a complex realisation $(G, p)$ of a graph $G=(V, E)$, its rigidity matroid $\mathcal{R}(G, p)=(E, \mathcal{I})$ is defined by taking $\mathcal{I}$ to be the family of all subsets of $E$ which correspond to linearly independent sets of rows in the rigidity matrix of $(G, p)$. It is not difficult to see that the set of independent subsets of $E$ is 
the same for all generic complex realisations of $G$. We refer to the resulting matroid as the rigidity matroid of $G$ and denote it by $\mathcal{R}(G)$.

Given a matroid $\mathcal{M}=(E, \mathcal{I})$ we may define an equivalence relation on $E$ by saying that $e, f \in E$ are related if $e=f$ or if there is a circuit, i.e. minimal dependent set, $C$ of $\mathcal{M}$ with $e, f \in C$. The equivalence classes are called the components of $\mathcal{M}$. If $\mathcal{M}$ has at least two elements and only one component then $\mathcal{M}$ is said to be connected. We say that a graph $G=(V, E)$ is $\mathcal{M}$ connected if its rigidity matroid $\mathcal{R}(G)$ is connected. The $\mathcal{M}$-components of $G$ are the subgraphs of $G$ induced by the components of $\mathcal{R}(G)$. For more examples and basic properties of $\mathcal{M}$-connected graphs see [16]. An efficient algorithm for constructing the $\mathcal{M}$-components of a graph is given in [3].

Theorem 5.3 Let $G=(V, E)$ be a an $\mathcal{M}$-connected graph and $u, v \in V$. Then $\{u, v\}$ is globally linked in $G$ if and only if $u$ and $v$ are joined by three internally disjoint paths in $G$.

Proof. Necessity follows for real (and hence also complex) generic realisations by [17, Lemma 5.6]. Sufficiency follows by applying the same proof technique as for [17, Theorem 5.7] but using Theorem 5.2 in place of [17, Theorem 4.2]

The following conjecture is a complex version of [17, Conjecture 5.9]. It would characterise when two vertices in a rigid graph are globally linked.

Conjecture 5.4 Let $G=(V, E)$ be a rigid graph and $u, v \in V$. Then $\{u, v\}$ is globally linked in $G$ if and only if either $u v \in E$ or $u$ and $v$ are joined by three internally disjoint paths in some $\mathcal{M}$-connected component of $G$.

Note that the 'sufficiency part' of Conjecture 5.4 follows from Theorem 5.3.

\section{Separable graphs}

A $k$-separation of a graph $G=(V, E)$ is a pair $\left(G_{1}, G_{2}\right)$ of edge-disjoint subgraphs of $G$ each with at least $k+1$ vertices such that $G=G_{1} \cup G_{2}$ and $\left|V\left(G_{1}\right) \cap V\left(G_{2}\right)\right|=k$. If $\left(G_{1}, G_{2}\right)$ is a $k$-separation of $G$, then we say that $G$ is $k$-separable and that $V\left(G_{1}\right) \cap V\left(G_{2}\right)$ is a $k$-separator of $G$. We will obtain expressions for $c(G)$ when $G$ is a rigid graph with a 2-separation, and also when $G$ has a 3 -separation induced by a 3 -edge-cut. 
Lemma 6.1 Let $\left(G_{1}, G_{2}\right)$ be a 2-separation of a rigid graph $G$ with $V\left(G_{1}\right) \cap$ $V\left(G_{2}\right)=\left\{v_{1}, v_{2}\right\}$ and let $H_{i}=G_{i}+e$ where $e=v_{1} v_{2}$ for $i=1,2$. Suppose that $\left\{v_{1}, v_{2}\right\}$ is globally linked in $G$. Then $c(G)=2 c\left(H_{1}\right) c\left(H_{2}\right)$.

Proof. Let $(G, p)$ be a generic realisation of $G$ and choose $d_{0} \in \mathbb{C}$ with $d\left(p\left(v_{1}\right)-p\left(v_{2}\right)\right)=d_{0}^{2}$ and $\operatorname{Arg} d_{0} \in(0, \pi]$. Let $S$ be the set of all realisations $(G, q)$ which are equivalent to $(G, p)$ and satisfy $q\left(v_{1}\right)=(0,0)$ and $q\left(v_{2}\right)=$ $\left(0, d_{0}\right)$. Lemma 3.1 and the hypothesis that $\left\{v_{1}, v_{2}\right\}$ is globally linked in $G$ imply that $|S|=2 c(G)$.

For $i=1,2$, the hypothesis that $G$ is rigid implies that $H_{i}$ is rigid. Let $S_{i}$ be the set of all realisations $\left(H_{i}, q_{i}\right)$ which are equivalent to $\left(H_{i},\left.p\right|_{H_{i}}\right)$ and satisfy $q_{i}\left(v_{1}\right)=(0,0)$ and $q_{i}\left(v_{2}\right)=\left(0, d_{0}\right)$. Lemma 3.1 and the fact that $v_{1} v_{2} \in E\left(H_{i}\right)$ imply that $\left|S_{i}\right|=2 c\left(H_{i}\right)$. It is straightforward to check that the map $\theta: S \rightarrow S_{1} \times S_{2}$ defined by $\theta(G, q)=\left[\left(H_{1},\left.q\right|_{V\left(H_{1}\right.}\right),\left(H_{2},\left.q\right|_{V\left(H_{2}\right.}\right)\right]$ is a bijection. Hence $2 c(G)=|S|=\left|S_{1}\right| \times\left|S_{2}\right|=4 c\left(H_{1}\right) c\left(H_{2}\right)$.

We next show that we can apply Lemma 6.1 when $G$ has a 2 -separation $\left(G_{1}, G_{2}\right)$ in which $G_{1}$ and $G_{2}$ are both rigid. We need one more piece of matroid terminology. An $\mathcal{M}$-circuit in a graph $G$ is a subgraph $H$ such that $E(H)$ is a circuit in the rigidity matroid of $G$.

Lemma 6.2 Let $\left(G_{1}, G_{2}\right)$ be a 2-separation of a rigid graph $G$ with $V\left(G_{1}\right) \cap$ $V\left(G_{2}\right)=\left\{v_{1}, v_{2}\right\}$ and let $H_{i}=G_{i}+e$ where $e=v_{1} v_{2}$ for $i=1,2$. Suppose that $G_{1}$ and $G_{2}$ are both rigid. Then $\{u, v\}$ is globally linked in $G$ and $c(G)=$ $2 c\left(H_{1}\right) c\left(H_{2}\right)$.

Proof. We first show that $\left\{v_{1}, v_{2}\right\}$ is globally linked in $G$. This holds trivially if $e \in E(G)$ and hence we may suppose that $e \notin E(G)$. Since $G_{i}$ is rigid, $e_{i}$ is contained in an $\mathcal{M}$-circuit $C_{i}$ of $H_{i}$ for each $i=1,2$. Then $C=\left(C_{1}-e\right) \cup\left(C_{2}-e\right)$ is an $\mathcal{M}$-circuit of $G$ by [2, Lemma 4.1]. We may now use Theorem 5.3 to deduce that $\{u, v\}$ is globally linked in $C$. Since $C \subseteq G$, $\{u, v\}$ is globally linked in $G$. The fact that $c(G)=2 c\left(H_{1}\right) c\left(H_{2}\right)$ now follows immediately from Lemma 6.1.

In order to obtain results for graphs with 2-separations $\left(G_{1}, G_{2}\right)$ in which $G_{1}$ and $G_{2}$ are not both rigid, we need a result concerning the number of complex realisations of a rigid graph satisfying given 'distance' constraints. 
Lemma 6.3 Let $G=(V, E)$ be a rigid graph with $V=\left\{v_{1}, v_{2}, \ldots, v_{n}\right\}$, $E=\left\{e_{1}, e_{2}, \ldots, e_{m}\right\}$ and $e_{i}=v_{i_{1}} v_{i_{2}}$ for all $1 \leq i \leq m$. Suppose that $T=$ $\left\{e_{1}, e_{2}, \ldots, e_{t}\right\} \subseteq E$ is such that $\operatorname{rank}(G-T)=\operatorname{rank}(G)-t . \quad$ Let $(G, p)$ be a generic realisation of $G$ and $d_{T}^{*}=\left\{d_{1}^{*}, d_{2}^{*}, \ldots, d_{t}^{*}\right\} \subset \mathbb{C}$ be algebraically independent over $\mathbb{Q}\left(d_{G-T}(p)\right)$. Then the number of pairwise non-congruent realisations $(G, q)$ of $G$ with $(G-T, q)$ equivalent to $(G-T, p)$ and $d\left(p\left(v_{i_{1}}\right)-\right.$ $\left.p\left(v_{i_{2}}\right)\right)=d_{i}^{*}$ for all $e_{i} \in T$ is $c(G)$.

Proof. Let $K=\mathbb{Q}\left(d_{G-T}(p)\right)$. We will define polynomials $f_{i} \in K[X, Y, D]$ for $1 \leq i \leq m$, where $X=\left(X_{1}, X_{2}, \ldots, X_{n}\right), Y=\left(Y_{1}, Y_{2}, \ldots, Y_{n}\right)$, and $D=\left(D_{1}, D_{2}, \ldots, D_{t}\right)$ are indeterminates. We first associate two variables $X_{i}, Y_{i}$ with each $v_{i} \in V$ and a variable $D_{i}$ with each $e_{i} \in T$. We then put $f_{i}=\left(X_{i_{1}}-X_{i_{2}}\right)^{2}+\left(Y_{i_{1}}-Y_{i_{2}}\right)^{2}-D_{i}$ for each $e_{i} \in T$ and $f_{i}=\left(X_{i_{1}}-X_{i_{2}}\right)^{2}+$ $\left(Y_{i_{1}}-Y_{i_{2}}\right)^{2}-d\left(p\left(v_{i_{1}}\right)-p\left(v_{i_{2}}\right)\right)$ for each $e_{i} \in E \backslash T$.

We now apply Lemma 3.3. We need to find $x, y \in \mathbb{C}^{n}$ and $d \in \mathbb{C}^{t}$ such that $f_{i}(x, y, d)=0$ for all $1 \leq i \leq m$, and $\operatorname{td}[K(d), K]=t$. This is easy since we can just put $\left(x_{i}, y_{i}\right)=p\left(v_{i}\right)$ for all $v_{i} \in V$ and $d_{i}=d\left(p\left(v_{i_{1}}\right)-p\left(v_{i_{2}}\right)\right)$ for all $e_{i} \in T$, and use the definition of the polynomials $f_{i}$ to deduce that $f_{i}(x, y, d)=0$ for all $1 \leq i \leq m$. Since $G$ is rigid $\operatorname{td}\left[\mathbb{Q}\left(d_{G}(p)\right), \mathbb{Q}\right]=2 n-3$ and $\operatorname{td}\left[\mathbb{Q}\left(d_{G-T}(p)\right), \mathbb{Q}\right]=\operatorname{rank}(G-T)=2 n-3-t$ by Lemma 3.4. Since $\operatorname{td}\left[\mathbb{Q}\left(d_{G}(p)\right), \mathbb{Q}\right]=\operatorname{td}[K(d), K]+\operatorname{td}[K, \mathbb{Q}]$ we have $\operatorname{td}[K(d), K]=t$. Since we also have $\operatorname{td}\left[K\left(d_{T}^{*}\right), K\right]=t$, Lemma 3.3 implies that there exists a realisation $(G, q)$ with $(G-T, q)$ equivalent to $(G-T, p)$ and $d\left(p\left(v_{i_{1}}\right)-p\left(v_{i_{2}}\right)\right)=d_{i}^{*}$ for all $e_{i} \in T$.

We may assume that $(G, q)$ is in canonical position with respect to $v_{1}, v_{2}, v_{3}$. Since $\mathbb{Q}\left(d_{T}^{*}\right) \subseteq \mathbb{Q}\left(d_{G}(q)\right), \operatorname{td}\left[\mathbb{Q}\left(d_{G}(q)\right): \mathbb{Q}\right]=\operatorname{td}\left[\mathbb{Q}\left(d_{G}(q)\right): K\right]+\operatorname{td}[K: \mathbb{Q}] \geq$ $\operatorname{td}\left[\mathbb{Q}\left(d_{T}^{*}\right): K\right]+\operatorname{td}[K: \mathbb{Q}] \geq|T|+2 n-3-|T|=2 n-3$. Since $(G, q)$ is in canonical position and $\mathbb{Q}\left(d_{G}(q)\right) \subseteq \mathbb{Q}(q)$ we must have $\operatorname{td}[\mathbb{Q}(q): \mathbb{Q}]=2 n-3$. We may now rotate and translate $(G, q)$ to obtain a generic framework. Hence $(G, q)$ is quasi-generic and and the number of pairwise non-congruent realisations of $G$ which are equivalent to $(G, q)$ is $c(G)$.

Our next result is needed to enable us to apply Lemma 6.3 to $k$-separations.

Lemma 6.4 Let $H_{1}, H_{2}$ be rigid graphs. Put $H=H_{1} \cup H_{2}, H_{3}=H_{1} \cap H_{2}$, and $T=E\left(H_{3}\right)$. Suppose that $H_{3}$ is isostatic and that $\operatorname{rank}\left(H_{2}-T\right)=$ $\operatorname{rank}\left(H_{2}\right)-|T|$. Let $(H, p)$ be a quasi-generic realisation of $H, G_{1}$ be a spanning rigid subgraph of $H_{1}$, and $\left(G_{1}, q_{1}\right)$ be a realisation of $G_{1}$ which 
is equivalent to $\left(G_{1},\left.p\right|_{G_{1}}\right)$. Then $d_{T}^{*}=\left\{d\left(q_{1}(u)-q_{1}(v)\right): u v \in T\right\}$ is algebraically independent over $\mathbb{Q}\left(d_{H_{2}-T}\left(\left.p\right|_{H_{2}}\right)\right)$.

Proof. If $T=\emptyset$ there is nothing to prove so we may suppose that $|T| \geq 1$ and hence $\left|V\left(H_{3}\right)\right| \geq 2$. We may also assume that $(H, p)$ and $\left(H_{1}, q_{1}\right)$ are both in canonical position with $p(u)=(0,0)=q_{1}(u), p(v)=(0, y)$ and $q_{1}(v)=(0, z)$ for some $y, z \in \mathbb{C}$ and some $u, v \in V\left(H_{3}\right)$.

Since $H_{1}, H_{2}$ are rigid, $H=H_{1} \cup H_{2}$ is rigid. Let $F$ be a spanning isostatic subgraph of $H$ which contains $T$ and let $F_{i}=F \cap H_{i}$. Then

$$
\begin{aligned}
|E(F)| & =\left|E\left(F_{1}\right)\right|+\left|E\left(F_{2}\right)\right|-|T| \\
& \leq\left(2\left|V\left(H_{1}\right)\right|-3\right)+2\left(\left|V\left(H_{2}\right)\right|-3\right)-2\left(\left|V\left(H_{3}\right)\right|-3\right) \\
& =2|V(H)|-3 .
\end{aligned}
$$

Equality must occur throughout and hence $F_{i}$ is a spanning isostatic subgraph of $H_{i}$ for $i=1,2$. Lemma 3.4 now implies that

$$
\overline{\mathbb{Q}\left(d_{F_{1}}\left(q_{1}\right)\right)}=\overline{\mathbb{Q}\left(q_{1}\right)}=\overline{\mathbb{Q}\left(d_{G_{1}}\left(q_{1}\right)\right)}
$$

and

$$
\overline{\mathbb{Q}\left(d_{F_{1}}(p)\right)}=\overline{\mathbb{Q}(p)}=\overline{\left.\mathbb{Q}\left(d_{H_{1}}(p)\right)\right)}=\overline{\left.\mathbb{Q}\left(d_{G_{1}}(p)\right)\right)} .
$$

Since $\left(G_{1}, q_{1}\right)$ and $\left(G_{1},\left.p\right|_{G_{1}}\right)$ are equivalent $d_{G_{1}}\left(q_{1}\right)=d_{G_{1}}(p)$. Equations $(8)$ and (9) now give $\overline{\mathbb{Q}\left(d_{F_{1}}(p)\right)}=\overline{\mathbb{Q}\left(d_{F_{1}}\left(q_{1}\right)\right)}$ and hence

$$
\begin{aligned}
\overline{\mathbb{Q}\left(d_{H}(p)\right)} & =\overline{\mathbb{Q}\left(d_{H_{1}}(p), d_{H_{2}-T}(p)\right)} \\
& =\overline{\mathbb{Q}\left(d_{F_{1}}(p), d_{H_{2}-T}(p)\right)} \\
& =\overline{\mathbb{Q}\left(d_{F_{1}}\left(q_{1}\right), d_{H_{2}-T}(p)\right)} .
\end{aligned}
$$

Thus

$$
\operatorname{td}\left[\mathbb{Q}\left(d_{H}(p)\right): \mathbb{Q}\right]=\operatorname{td}\left[\mathbb{Q}\left(d_{H_{2}-T}(p): \mathbb{Q}\right]+\operatorname{td}\left[\mathbb { Q } \left(d_{F_{1}}\left(q_{1}\right): \mathbb{Q}\left(d_{H_{2}-T}(p)\right] .\right.\right.\right.
$$

By Lemma 3.4, $\operatorname{td}\left[\mathbb{Q}\left(d_{H}(p)\right): \mathbb{Q}\right]=\operatorname{rank}(H)=2|V(H)|-3$ and

$$
\operatorname{td}\left[\mathbb{Q}\left(d_{H_{2}-T}(p)\right): \mathbb{Q}\right]=\operatorname{rank}\left(H_{2}-T\right)=2\left|V\left(H_{2}\right)\right|-3-|T| .
$$

Thus

$$
\begin{aligned}
\operatorname{td}\left[\mathbb { Q } \left(d_{F_{1}}\left(q_{1}\right): \mathbb{Q}\left(d_{H_{2}-T}(p)\right]\right.\right. & =2|V(H)|-3-\left(2\left|V\left(H_{2}\right)\right|-3-|T|\right) \\
& =2\left|V\left(F_{1}\right)\right|-3=\left|E\left(F_{1}\right)\right| .
\end{aligned}
$$


Hence $d_{F_{1}}\left(q_{1}\right)$ is algebraically independent over $\mathbb{Q}\left(d_{H_{2}-T}(p)\right.$. Since $T \subseteq$ $E\left(F_{1}\right), d_{T}^{*}$ is also algebraically independent over $\mathbb{Q}\left(d_{H_{2}-T}(p)\right.$.

Lemma 6.5 Let $\left(G_{1}, G_{2}\right)$ be a 2-separation of a rigid graph $G$ with $V\left(G_{1}\right) \cap$ $V\left(G_{2}\right)=\left\{v_{1}, v_{2}\right\}$. Suppose that $G_{2}$ is not rigid and put $H_{2}=G_{2}+e$ where $e=v_{1} v_{2}$. Then $G_{1}$ and $H_{2}$ are both rigid and $c(G)=2 c\left(G_{1}\right) c\left(H_{2}\right)$.

Proof. Let $F$ be a spanning isostatic subgraph of $G$. We have $\mid E(F) \cap$ $E\left(G_{1}\right)|\leq 2| V\left(G_{1}\right) \mid-3$, and $\left|E(F) \cap E\left(G_{2}\right)\right| \leq 2\left|V\left(G_{2}\right)\right|-4$ since $G_{2}$ is not rigid. Thus

$$
\begin{aligned}
|E(F)| & =\left|E(F) \cap E\left(G_{1}\right)\right|+\left|E(F) \cap E\left(G_{2}\right)\right| \\
& \leq 2\left|V\left(G_{1}\right)\right|-3+2\left|V\left(G_{2}\right)\right|-4=2|V(F)|-3 .
\end{aligned}
$$

Since $F$ is rigid, we must have equality throughout. In particular $\mid E(F) \cap$ $E\left(G_{1}\right)|=2| V\left(G_{1}\right) \mid-3$ so $G_{1}$ is rigid.

Consider the 2-separation $\left(G_{1}, H_{2}\right)$ of $H=G+e$, and let $F^{\prime}$ be a spanning isostatic subgraph of $H$ which contains $e$. Then $\left|E\left(F^{\prime}\right) \cap E\left(H_{2}\right)\right| \leq 2\left|V\left(H_{2}\right)\right|-$ 3 and, since $e \in E\left(F^{\prime}\right),\left|E\left(F^{\prime}\right) \cap E\left(G_{1}\right)\right| \leq 2\left|V\left(G_{1}\right)\right|-4$. Thus

$$
\begin{aligned}
\left|E\left(F^{\prime}\right)\right| & =\left|E\left(F^{\prime}\right) \cap E\left(G_{1}\right)\right|+\left|E\left(F^{\prime}\right) \cap E\left(H_{2}\right)\right| \\
& \leq 2\left|V\left(G_{1}\right)\right|-4+2\left|V\left(H_{2}\right)\right|-3=2\left|V\left(F^{\prime}\right)\right|-3
\end{aligned}
$$

Since $F^{\prime}$ is rigid, we must have equality throughout. In particular $\mid E\left(F^{\prime}\right) \cap$ $E\left(H_{2}\right)|=2| V\left(H_{2}\right) \mid-3$ so $H_{2}$ is rigid.

Let $(G, p)$ be a generic realisation of $G$. For each $z \in \mathbb{C} \backslash\{0\}$ with $\operatorname{Arg} z \in(0, \pi]$ let $S(z)$ be the set of all realisations $(G, q)$ of $G$ such that $(G, q)$ is equivalent to $(G, p), q\left(v_{1}\right)=(0,0)$ and $q\left(v_{2}\right)=(0, z)$. Define $S_{1}(z)$ and $S_{2}(z)$ similarly by replacing $(G, p)$ by $\left(G_{1},\left.p\right|_{G_{1}}\right)$ and $\left(H_{2},\left.p\right|_{H_{2}}\right)$ respectively. Lemma 3.1 and Theorem 3.6 imply that $S(z), S_{1}(z)$ and $S_{2}(z)$ are finite, and are non-empty for only finitely many values of $z$. In addition we have

$$
2 c(G)=\sum_{S(z) \neq \emptyset}|S(z)| \text { and } 2 c\left(G_{1}\right)=\sum_{S_{1}(z) \neq \emptyset}\left|S_{1}(z)\right| .
$$

We will show that

$$
|S(z)|=2\left|S_{1}(z)\right| c\left(H_{2}\right)
$$


for all $z \in \mathbb{C} \backslash\{0\}$ with $\operatorname{Arg} z \in(0, \pi]$. If $S_{1}(z)=\emptyset$ then we must also have $S(z)=\emptyset$, since for any $(G, q) \in S(z)$ we would have $\left.\left(G_{1},\left.q\right|_{V\left(G_{1}\right.}\right)\right) \in S_{1}(z)$, so (11) holds trivially.

We next consider the case when $S_{1}(z) \neq \emptyset$. Choose $\left(G_{1}, q_{1}\right) \in S_{1}(z)$. We may apply Lemma 6.4 with $H=G+e, H_{1}=G_{1}+e, T=\{e\}$ and $d_{T}^{*}=\left\{d\left(q_{1}\left(v_{1}\right)-q_{1}\left(v_{2}\right)\right)\right\}$ to deduce that $d_{T}^{*}$ is algebraically independent over $\mathbb{Q}\left(d_{H_{2}}(p)\right)$. We may then apply Lemma 6.3 (with $G=H_{2}$ ) and Lemma 3.1 to deduce that $\left|S_{2}(z)\right|=2 c\left(H_{2}\right)$. Since the map $\theta: S(z) \rightarrow S_{1}(z) \times S_{2}(z)$ by $\theta(G, q)=\left[\left(G_{1},\left.q\right|_{V\left(G_{1}\right)}\right),\left(H_{2},\left.q\right|_{V\left(G_{2}\right)}\right)\right]$ is a bijection, we have

$$
|S(z)|=\left|S_{1}(z)\right|\left|S_{2}(z)\right|=2\left|S_{1}(z)\right| c\left(H_{2}\right) .
$$

Thus (11) also holds when $S_{1}(z) \neq \emptyset$.

Equation (11) and the fact that $c\left(H_{2}\right) \neq 0$ imply that $S_{1}(z)=\emptyset$ if and only if $S(z)=\emptyset$. We can now use equations (10) and (11) to deduce that

$$
c(G)=\sum_{S(z) \neq \emptyset}|S(z)|=2 \sum_{S_{1}(z) \neq \emptyset}\left|S_{1}(z)\right| c\left(H_{2}\right)=2 c\left(G_{1}\right) c\left(H_{2}\right) .
$$

Note that Lemma 4.1 is the special case of Lemma 6.5 when $G_{2}$ is a path of length two.

Lemmas 6.1 and 6.5 immediately give

Theorem 6.6 Suppose that $G$ is a rigid graph and $\left(G_{1}, G_{2}\right)$ is a 2-separation of $G$ with $V\left(G_{1}\right) \cap V\left(G_{2}\right)=\{u, v\}$. Then $G_{1}+u v, G_{2}+u v$, and at least one of $G_{1}, G_{2}$ are rigid. Furthermore:

(a) if $G_{1}$ and $G_{2}$ are both rigid then $c(G)=2 c\left(G_{1}+u v\right) c\left(G_{2}+u v\right)$;

(b) if $G_{1}$ is rigid and $G_{2}$ is not rigid then $c(G)=2 c\left(G_{1}\right) c\left(G_{2}+u v\right)$.

We next state a complementary result for $k$-separations when $k \geq 3$ and the common intersection is globally rigid. Its proof is straightforward.

Theorem 6.7 Suppose that $G$ is a rigid graph and $\left(G_{1}, G_{2}\right)$ is a k-separation of $G$ such that $k \geq 3$ and $G_{1} \cap G_{2}$ is globally rigid. Then $c(G)=c\left(G_{1}\right) c\left(G_{2}\right)$.

We close this section by deriving a reduction formula for $c(G)$ when $G$ has a 3-edge-cut. We first need to determine $c(G)$ when $G$ is the triangular prism i.e. the graph on six vertices consisting of two disjoint triangles joined by a perfect matching shown in Figure 3. 


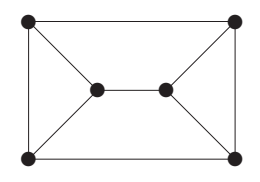

Figure 3: The triangular prism.

Lemma 6.8 Let $P$ be the triangular prism. Then $c(P)=12$.

Proof. It is well known that every realisation of $P$ in $\mathbb{C}^{2}$ has at most 12 equivalent, non-congruent realisations and that there exists a (real) realisation $(G, p)$ with 12 equivalent, non-congruent (real) realisations in which the vertices are not collinear, see for example $[4,8]$. We can now use Theorem 3.7 to deduce that $c(G)=12$.

Theorem 6.9 Suppose that $G$ is a rigid graph and $G=G_{1} \cup G_{2} \cup\left\{e_{1}, e_{2}, e_{3}\right\}$ where $V\left(G_{1}\right) \cap V\left(G_{2}\right)=\emptyset, e_{i}=u_{i} v_{i}$ for $1 \leq i \leq 3, u_{1}, u_{2}, u_{3}$ are distinct vertices of $G_{1}$, and $v_{1}, v_{2}, v_{3}$ are distinct vertices of $G_{2}$. Then $G_{1}$ and $G_{2}$ are rigid and $c(G)=12 c\left(G_{1}\right) c\left(G_{2}\right)$.

Proof. Let $F$ be a spanning isostatic subgraph of $G$. We have $\mid E(F) \cap$ $E\left(G_{1}\right)|\leq 2| V\left(G_{1}\right) \mid-3$ and $\left|E(F) \cap E\left(G_{2}\right)\right| \leq 2\left|V\left(G_{2}\right)\right|-3$. Thus

$$
\begin{aligned}
|E(F)| & \leq\left|E(F) \cap E\left(G_{1}\right)\right|+\left|E(F) \cap E\left(G_{2}\right)\right|+3 \\
& \leq 2\left|V\left(G_{1}\right)\right|-3+2\left|V\left(G_{2}\right)\right|-3+3=2|V(F)|-3 .
\end{aligned}
$$

Since $F$ is rigid, we must have equality throughout. In particular $\mid E(F) \cap$ $E\left(G_{i}\right)|=2| V\left(G_{i}\right) \mid-3$ so $G_{i}$ is rigid for $i=1,2$.

Claim 1 Let $H_{2}$ be obtained from $G_{2}$ by adding the vertices $u_{1}, u_{2}, u_{3}$ and edges $u_{1} u_{2}, u_{2} u_{3}, u_{3} u_{1}, u_{1} v_{1}, u_{2} v_{2}, u_{3} v_{3}$. Then $c(G)=c\left(G_{1}\right) c\left(H_{2}\right)$.

Proof. Let $(G, p)$ be a generic realisation of $G$. For each fixed $b_{2}, a_{3}, b_{3} \in$ $\mathbb{C} \backslash\{0\}$ with Arg $b_{2}, \operatorname{Arg} a_{3} \in(0, \pi]$ let $S\left(b_{2}, a_{3}, b_{3}\right)$ be the set of all realisations 
$(G, q)$ of $G$ such that $(G, q)$ is equivalent to $(G, p), q\left(u_{1}\right)=(0,0), q\left(u_{2}\right)=$ $\left(0, b_{2}\right)$ and $q\left(u_{3}\right)=\left(a_{3}, b_{3}\right)$. Define $S_{1}\left(b_{2}, a_{3}, b_{3}\right)$ and $S_{2}\left(b_{2}, a_{3}, b_{3}\right)$ similarly by replacing $(G, p)$ by $\left(G_{1},\left.p\right|_{G_{1}}\right)$ and $\left(H_{2},\left.p\right|_{H_{2}}\right)$ respectively. Lemma 3.1 and Theorem 3.6 imply that $S\left(b_{2}, a_{3}, b_{3}\right), S_{1}\left(b_{2}, a_{3}, b_{3}\right)$ and $S_{2}\left(b_{2}, a_{3}, b_{3}\right)$ are finite, and are non-empty for only finitely many values of $b_{2}, a_{3}, b_{3}$. In addition we have

$$
c(G)=\sum_{S\left(b_{2}, a_{3}, b_{3}\right) \neq \emptyset}\left|S\left(b_{2}, a_{3}, b_{3}\right)\right| \text { and } c\left(G_{1}\right)=\sum_{S_{1}\left(b_{2}, a_{3}, b_{3}\right) \neq \emptyset}\left|S_{1}\left(b_{2}, a_{3}, b_{3}\right)\right| .
$$

We will show that

$$
\left|S\left(b_{2}, a_{3}, b_{3}\right)\right|=\left|S_{1}\left(b_{2}, a_{3}, b_{3}\right)\right| c\left(H_{2}\right)
$$

for all $b_{2}, a_{3}, b_{3} \in \mathbb{C} \backslash\{0\}$ with $\operatorname{Arg} b_{2}, \operatorname{Arg} a_{3} \in(0, \pi]$. If $S_{1}\left(b_{2}, a_{3}, b_{3}\right)=\emptyset$ then we must also have $S\left(b_{2}, a_{3}, b_{3}\right)=\emptyset$, since for any $(G, q) \in S\left(b_{2}, a_{3}, b_{3}\right)$ we would have $\left.\left(G_{1},\left.q\right|_{V\left(G_{1}\right.}\right)\right) \in S_{1}\left(b_{2}, a_{3}, b_{3}\right)$, so (13) holds trivially.

We next consider the case when $S_{1}\left(b_{2}, a_{3}, b_{3}\right) \neq \emptyset$. Choose $\left(G_{1}, q_{1}\right) \in$ $S_{1}\left(b_{2}, a_{3}, b_{3}\right)$. Let $T=\left\{u_{1} u_{2}, u_{2} u_{3}, u_{3} u_{1}\right\}$ and $d_{T}^{*}=\left\{d\left(q_{1}\left(u_{i}\right)-q_{1}\left(u_{j}\right)\right)\right.$ : $\left.u_{i} u_{j} \in T\right\}$. We may apply Lemma 6.4 with $(H, p)=(G \cup T, p)$ and $\left(H_{1}, q_{1}\right)=$ $\left(G_{1} \cup T, q_{1}\right)$ to deduce that $d_{T}^{*}$ is algebraically independent over $\mathbb{Q}\left(d_{H_{2}-T}(p)\right)$. We may then apply Lemma 6.3 (with $G=H_{2}$ ) to deduce that $\left|S_{2}\left(b_{2}, a_{3}, b_{3}\right)\right|=$ $c\left(H_{2}\right)$. Since the map $\theta: S\left(b_{2}, a_{3}, b_{3}\right) \rightarrow S_{1}\left(b_{2}, a_{3}, b_{3}\right) \times S_{2}\left(b_{2}, a_{3}, b_{3}\right)$ by $\theta(G, q)=\left[\left(G_{1},\left.q\right|_{V\left(G_{1}\right)}\right),\left(G_{2},\left.q\right|_{V\left(G_{2}\right)}\right)\right]$ is a bijection, we have

$$
\left|S\left(b_{2}, a_{3}, b_{3}\right)\right|=\left|S_{1}\left(b_{2}, a_{3}, b_{3}\right)\right|\left|S_{2}\left(b_{2}, a_{3}, b_{3}\right)\right|=\left|S_{1}\left(b_{2}, a_{3}, b_{3}\right)\right| c\left(H_{2}\right) .
$$

Thus (13) also holds when $S_{1}\left(b_{2}, a_{3}, b_{3}\right) \neq \emptyset$.

Equation (13) and the fact that $c\left(H_{2}\right) \neq 0$ imply that $S_{1}\left(b_{2}, a_{3}, b_{3}\right)=\emptyset$ if and only if $S\left(b_{2}, a_{3}, b_{3}\right)=\emptyset$. We can now use equations (12) and (13) to deduce that

$$
c(G)=\sum_{S\left(b_{2}, a_{3}, b_{3}\right) \neq \emptyset}\left|S\left(b_{2}, a_{3}, b_{3}\right)\right|=\sum_{S_{1}\left(b_{2}, a_{3}, b_{3}\right) \neq \emptyset}\left|S_{1}\left(b_{2}, a_{3}, b_{3}\right)\right| c\left(H_{2}\right)=c\left(G_{1}\right) c\left(H_{2}\right) .
$$

This completes the proof of Claim 1.

We may apply the argument of Claim 1 to $H_{2}$ to deduce that $c\left(H_{2}\right)=$ $c\left(G_{2}\right) c(P)$, where $P$ is the triangular prism. Claim 1 and the fact that $c(P)=12$ now give $c(G)=12 c\left(G_{1}\right) c\left(G_{2}\right)$. 


\section{Two families of graphs}

We use the results from the previous section to determine $c(G)$ for two important families of rigid graphs.

\section{Quadratically solvable graphs}

Let $G=(V, E)$ be an isostatic graph with $E=\left\{e_{1}, e_{2}, \ldots, e_{m}\right\}$ and $e_{i}=u_{i} v_{i}$ for $1 \leq i \leq m$. Then $G$ is quadratically solvable if for all $d=\left(d_{1}, d_{2}, \ldots, d_{m}\right) \in$ $\mathbb{C}^{m}$ such that $\left\{d_{1}, d_{2}, d_{3}, \ldots, d_{m}\right\}$ is algebraically independent over $\mathbb{Q}$, there exists a realisation $(G, p)$ of $G$ with $d\left(p\left(u_{i}\right)-p\left(v_{i}\right)\right)=d_{i}$ for all $1 \leq i \leq m$, in which $\mathbb{Q}(p)$ is contained in a quadratic extension of $\mathbb{Q}(d)$ i.e. there exists a sequence of field extensions $K_{1} \subset K_{2} \subset \ldots \subset K_{m}$ such that $K_{1}=\mathbb{Q}(d)$, $K_{m}=\mathbb{Q}(p)$ and $K_{i+1}=K_{i}(x)$ for some $x^{2} \in K_{i}$ for all $1 \leq i<m$. These graphs are important in the theory of equation solving in Computer Aided Design, see for example [11, 26].

We may recursively construct an infinite family $\mathcal{Q S}$ of quadratically solvable isostatic graphs as follows. We first put the complete graph on three vertices $K_{3}$ in $\mathcal{Q S}$. Then, for any two graphs $G_{1}, G_{2} \in \mathcal{Q S}$, any two vertices $u_{1}, v_{1}$ in $G_{1}$, and any edge $e=u_{2} v_{2}$ of $G_{2}$, we construct a new graph $G$ by 'gluing' $G_{1}$ and $G_{2}-e$ together along $u_{1}=u_{2}$ and $v_{1}=v_{2}$, and add $G$ to $\mathcal{Q S}$. Note that the special case of this construction when $G_{2}=K_{3}$ is just the Henneberg type 1 move and hence the family of graphs which can be constructed from $K_{3}$ by Henneberg type 1 moves is a subfamily of $\mathcal{Q S}$.

The second author conjectured in [26] that an isostatic graph $G$ is quadratically solvable if and only if it belongs to $\mathcal{Q S}$. This conjecture was subsequently verified for isostatic planar graphs in [27]. Our next result determines $c(G)$ for all $G \in \mathcal{Q S}$.

Theorem 7.1 Suppose $G \in \mathcal{Q S}$. Then $c(G)=2^{|V(G)|-3}$.

Proof. We use induction on $|V(G)|$. If $|V(G)|=3$ then $G=K_{3}$ and $c(G)=1$. Hence we may assume that $|V(G)|>3$. It follows from the recursive definition of $\mathcal{Q S}$ that there exists a 2-separation $\left(G_{1}, G_{2}\right)$ of $G$ with $V\left(G_{1}\right) \cap V\left(G_{2}\right)=\{u, v\}$ and such that $G_{1}$ and $G_{2}+u v$ both belong to $\mathcal{Q S}$. The theorem now follows from Lemma 6.5 and induction. 
We can use this result and the fact that all $\mathcal{Q S}$ graphs can be constructed using vertex splits to determine the maximum number of generic real realisations for any $\mathcal{Q S}$ graph. We will need a result from [27] that a graph $G$ is in $\mathcal{Q S}$ if and only if $G$ has a decomposition $G=G_{1} \cup G_{2} \cup G_{3}$ where $G_{1} \cap G_{2}=u_{3}, G_{2} \cap G_{3}=u_{1}, G_{3} \cap G_{1}=u_{2}$ and each $G_{i}$ is either $K_{2}$ or is in $\mathcal{Q S}$. We will also need the following concept: an edge $e$ in a $\mathcal{Q S}$ graph $G$ is contractible if either $G / e \in \mathcal{Q S}$ or $G / e=K_{2}$.

Lemma 7.2 Suppose that $G=(V, E) \in \mathcal{Q S}$ and $|V| \geq 3$. Then $G$ has at least two contractible edges.

Proof. Induction on $|V|$. If $|V|=3$ then $G=K_{3}$ and $G$ has three contractible edges. Hence we may suppose that $|V| \geq 4$. By [27], $G$ has a decomposition $G=G_{1} \cup G_{2} \cup G_{3}$ where $G_{1} \cap G_{2}=u_{3}, G_{2} \cap G_{3}=u_{1}$, $G_{3} \cap G_{1}=u_{2}$ and each $G_{i}$ is either $K_{2}$ or in $\mathcal{Q S}$. Since $|V| \geq 4$ we may assume that $G_{1} \neq K_{2}$. By induction, $G_{1}$ has two contractible edges $e$ and $f$. If neither $e$ nor $f$ is equal to $u_{2} u_{3}$ then they are both contractible in $G$ by [27] (since we have $G / e=G_{1} / e \cup G_{2} \cup G_{3}$ ). So suppose $e=u_{2} u_{3}$ and $f$ is contractible in $G$. If $\left|V\left(G_{2}\right)\right| \geq 3$ or $\left|V\left(G_{3}\right)\right| \geq 3$ then we can find another contractible edge in $G_{2}$ or $G_{3}$. Otherwise $G_{2}=K_{2}=G_{3}$ and the edges $u_{1} u_{3}$ and $u_{1} u_{2}$ are both contractible in $G$ (since $u_{2} u_{3} \in E$ and hence $\left.G / u_{1} u_{2}=G / u_{2} u_{3}=G_{1}\right)$.

Theorem 7.3 Suppose $G=(V, E) \in \mathcal{Q S}$. Then the maximum value of $r(G, p)$ over all generic real realisations $(G, p)$ of $G$ is $2^{|V|-3}$.

Proof. We use induction on $|V|$. If $|V(G)|=3$ then $G=K_{3}$ and and the theorem holds, so we may assume that $|V|>3$. Then $G$ has a contractible edge $e$ by Lemma 7.2. We can now use Theorem 4.8 and induction to deduce that $G$ has a generic real realisation $(G, p)$ such that $r(G, p) \geq 2^{|V|-3}$. On the other hand Theorem 7.1 shows that $r(G, q) \leq 2^{|V|-3}$ for all generic real realisations $(G, q)$.

Note that Theorem 7.3 extends the result of [4] that the maximum value of $r(G, p)$ over all generic realisations of graphs $G$ which can be constructed by Henneberg type 1 moves is $2^{|V|-3}$. 


\section{$\mathcal{M}$-connected graphs}

We will determine $c(G)$ when $G$ is an $\mathcal{M}$-connected graph. We need some new terminology. For each $\{u, v\} \subset V$, let $w_{G}(u, v)$ denote the number of connected components of $G-\{u, v\}$ and put $b(G)=\sum_{\{u, v\} \subset V}\left(w_{G}(u, v)-1\right)$. Note that $w_{G}(u, v)-1=0$ if $\{u, v\}$ is not a 2-separator of $G$, so we can assume that the summation in the definition of $b(G)$ is restricted to pairs $\{u, v\}$ which are 2-separators of $G$.

Theorem 7.4 Let $G$ be an $\mathcal{M}$-connected graph. Then $c(G)=2^{b(G)}$.

Proof. We use induction on $b(G)$. Suppose $b(G)=0$. Then $G$ is 3-connected and, since $G$ is $\mathcal{M}$-connected, it is also redundantly rigid. Hence $c(G)=1$ by Theorem 5.1 . Thus we may assume that $b(G) \geq 1$.

Choose vertices $u, v$ of $G$ with $w_{G}(u, v) \geq 2$ and let $\left(G_{1}, G_{2}\right)$ be a 2separation in $G$ with $V\left(G_{1}\right) \cap V\left(G_{2}\right)=\{u, v\}$. Let $H_{i}=G_{i}+u v$ for $i=1,2$. By [17, Lemma 5.3(b)], $H_{i}$ is $\mathcal{M}$-connected for $i=1,2$. In addition, [16, Lemma 3.6] implies that every 2-separator $\left\{u^{\prime}, v^{\prime}\right\}$ of $G$ which is distinct from $\{u, v\}$ is a 2-separator of $H_{i}$ for exactly one value of $i \in\{1,2\}$, and, for this value of $i$, satisfies $w_{G}\left(u^{\prime}, v^{\prime}\right)=w_{H_{i}}\left(u^{\prime}, v^{\prime}\right)$. Since we also have $w_{G}(u, v)=$

$w_{H_{1}}(u, v)+w_{H_{2}}(u, v)$, we may deduce that $b(G)=b\left(H_{1}\right)+b\left(H_{2}\right)-1$. Using induction and Lemma 6.1 we have

$$
c(G)=2 c\left(H_{1}\right) c\left(H_{2}\right)=2 \times 2^{b\left(H_{1}\right)} \times 2^{b\left(H_{2}\right)}=2^{b(G)} .
$$

Our expression for $c(G)$ in Theorem 7.4 is identical to that given for $r(G, p)$ in [17, Theorem 8.2] when $(G, p)$ is a generic real realisation of $G$, and provides an explanation for the fact that $r(G, p)$ is the same for all generic real realisations $(G, p)$ of an $\mathcal{M}$-connected graph $G$.

\section{Closing Remarks and Open Problems}

The obvious open problem is:

Problem 8.1 Can $c(G)$ be determined efficiently for an arbitrary rigid graph $G$ ? 


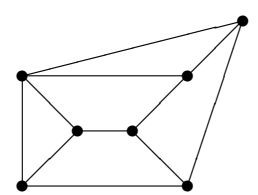

$G_{1}$

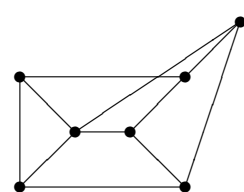

$G_{2}$

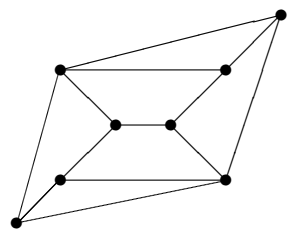

$G_{3}$

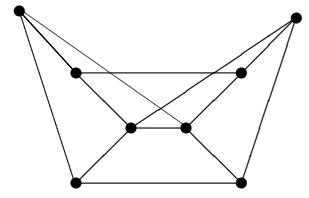

$G_{4}$

Figure 4: The graphs $G_{1}, G_{2}, G_{3}$ and $G_{4}$.

The isostatic graphs $G_{1}, G_{2}, G_{3}$ and $G_{4}$ of Figure 4 indicate that it may be difficult to obtain an affirmative answer to Problem 8.1 for all graphs. Emeris and Moroz [7] give a real framework $\left(G_{1}, p\right)$ with $r\left(G_{1}, p\right)=28$ and use mixed volume techniques to prove that $c\left(G_{1}, q\right) \leq 28$ for all complex rigid frameworks $\left(G_{1}, q\right)$, (an error in their proof was subsequently corrected in [8]). We may now use Theorem 3.7 to deduce that $c\left(G_{1}\right)=28$. A similar technique can be used to show that $c\left(G_{3}\right)=68$, see [19]. Computer calculations, i.e. calculating $c(G, p)$ for 'randomly chosen' realisations $(G, p)$, indicate that $c\left(G_{2}\right)=22$ and $c\left(G_{4}\right)=45$. These values have recently been confirmed by Josef Schicho (personal communication) using the algorithm described by Capco et al in [5]. It is difficult to imagine how these numbers could be deduced from the structures of $G_{1}, G_{2}, G_{3}$ and $G_{4}$.

Until recently, the fastest algorithms for determining $c(G)$ solved the associated system of polynomial equations using Gröbner basis calculations. Such algorithms are exponential and struggle to cope with some graphs on only seven vertices such as $G_{2}$. An exciting new algorithm based on a recurrence formula for $c(G)$ is described in [5]. Although still exponential, it has been used to determine $c(G)$ for all isostatic graphs on at most twelve vertices, see [5].

If we cannot determine $c(G)$ precisely then we could ask for tight asymptotic upper bounds on $c(G)$.

Problem 8.2 Determine the smallest $k \in \mathbb{R}$ such that $c(G)=O\left(k^{n}\right)$ for all rigid graphs $G$ with $n$ vertices. 
Clearly $c(G)$ will be maximised when $G$ is isostatic, and hence it follows from [4, Theorem 1.1] that $c(G) \leq \frac{1}{2}\left(\begin{array}{c}2 n-4 \\ n-2\end{array}\right) \approx 4^{n}$ for all rigid graphs $G$ with $n$ vertices. Borcea and Streinu [4, Proposition 5.6] also construct an infinite family of isostatic graphs $G$ with $c(G)=12^{(n-3) / 3} \approx 2.29^{n}$ by taking several copies of the triangular prism $P$ with a single triangle in common. The fact that $c(G)=12^{(n-3) / 3}$ for this family can be deduced from Lemmas 6.7 and 6.8. Emiris and Moroz [7] use a similar construction with $P$ replaced by $G_{1}$ to obtain an infinite family of isostatic graphs $G$ with $c(G)=28^{(n-3) / 4} \approx 2.3^{n}$. A similar construction based on $G_{3}$ gives an infinite family of isostatic graphs $G$ with $c(G)=68^{(n-3) / 5} \approx 2.33^{n}$.

The calculations in [5] determine the isostatic graphs on $n$ vertices which maximise $c(G)$ for all $n \leq 12$. The graphs for $n=6,7,8$ are the triangular prism $P, G_{1}$ and $G_{3}$. All three are planar graphs with exactly two triangles. The graphs from [5] for $n=9,10,11$ are also planar with exactly two triangles and the corresponding values for $c(G)$ are 172, 440 and 1144, respectively. (Curiously, their isostatic graph on $n=12$ vertices which maximises $c(G)$ has no triangles.) We may glue copies of their graph on 11 vertices together along a common triangle to obtain an infinite family of graphs with $c(G)=$ $1144^{(n-3) / 8} \approx 2.41^{n}$. It follows that the answer to Problem 8.2 will satisfy $1144^{1 / 8} \leq k \leq 4$.

It would also be of interest to determine a tight lower bound on $c(G)$ when $G$ is isostatic.

Conjecture 8.3 For all isostatic graphs $G$ with $n$ vertices, $c(G) \geq 2^{n-3}$.

Note that this conjecture holds with equality for $\mathcal{Q S}$ graphs by Theorem 7.1, and also holds for planar graphs by Theorem 4.9. The results of [5] confirm that the conjecture is also true when $n \leq 12$. Rather embarrassingly, the only lower bound we have for an arbitrary isostatic graph $G$ is the trivial bound $c(G) \geq 2$.

Since every isostatic graph can be obtained from a triangle by Henneberg type 1 and 2 moves, and since every Henneberg type 1 move doubles $c(G)$ by Lemma 4.1, it is tempting to try to prove Conjecture 8.3 by showing that if we perform the Henneberg type 2 move on a isostatic graph $G$ then we will increase $c(G)$ by at least a factor of two. Unfortunately this is not the case: the graph $G_{2}$ of Figure 4 can be obtained from the triangular prism $P$ by a Henneberg type 2 move; we have $c(P)=12$ and we have $c\left(G_{2}\right)=22<2 c(P)=24$. 
We may also consider the problem of deciding which graphs have rigid realisations in $\mathbb{C}^{2}$.

Conjecture 8.4 A graph $G=(V, E)$ has a realisation in $\mathbb{C}^{2}$ which is rigid and has $d(p(u)-p(v)) \neq 0$ for some $u v \in E$ if and only if $G$ is generically rigid.

It is straightforward to show that $G$ has a realisation in $\mathbb{C}^{2}$ which is rigid (and has $d(p(u)-p(v))=0$ for all $u v \in E$ ) if and only if $G$ is connected.

Our final problem is to consider the relationship between $c(G)$ and $r(G, p)$. Dylan Thurston asked whether every rigid graph $G$ has a generic real realisation $(G, p)$ such that $r(G, p)=c(G)$ at a workshop on global rigidity held at Cornell University in February 2011.

The graph $G_{4}$ in Figure 4 shows that the answer to this question is negative. We have $c\left(G_{4}\right)=45$. On the other hand, the proof technique used by Hendrickson [14] to obtain necessary conditions for global rigidity can be adapted to show that $r(G, p)$ is even for all generic real realisations of a graph $G$ which is rigid but not globally rigid. ${ }^{3}$ Thus $r\left(G_{4}, p\right) \leq 44$ for all generic real realisations of $G_{4}$. By glueing several copies of $G_{4}$ along a common edge, we may construct an infinite family of rigid graphs $G$ on $n$ vertices such that $r(G, p) / c(G) \leq(44 / 45)^{\frac{n-2}{6}}$ for all generic real realisations of $G$. It follows that we can make the ratio $r(G, p) / c(G)$ arbitrarily close to zero.

We may say a bit more about the parity argument used in the above construction. Let $G=(V, E)$ be a graph which is rigid but not globally rigid and $S$ be the set of all realisations which are in canonical position with respect to three given vertices $v_{1}, v_{2}, v_{3}$ and are equivalent to a given generic real realisation $(G, p)$ of $G$. Since all edge lengths in $(G, p)$ are real, the map $(G, q) \mapsto\left(G, q^{*}\right)$, where $q^{*}$ is obtained by taking the complex conjugates of the coordinates of $q$ and then, if necessary, reflecting the resulting framework in the axes to return to canonical position, is an involution on $S$.

\footnotetext{
${ }^{3}$ Let $S$ be the set of all real realisations which are in canonical position and are equivalent to $(G, p)$. If $G$ is not redundantly rigid then $G-e$ is not rigid for some edge $e$. In this case each component of the real configuration space of $(G-e, p)$ will contain an even number of elements of $S$. If $G$ is redundantly rigid then, since $G$ is not globally rigid, $G$ has a 2-separation. In this case reflecting one of the sides of the 2-separation in the line through the two vertices of the corresponding 2-separator gives an involution on $S$ with no fixed points.
} 
Suppose $\left(G, q^{*}\right)$ is equal to $(G, q)$ and let $q\left(v_{1}\right)=(0,0), q\left(v_{2}\right)=\left(0, y_{2}\right)$ and $q\left(v_{3}\right)=\left(x_{3}, y_{3}\right)$. Then $q^{*}\left(v_{2}\right)=\left(0, \pm \bar{y}_{2}\right)=\left(0, y_{2}\right)$. Hence $y_{2}$ is either real or imaginary.

We first consider the case when $y_{2}$ is real. We have $q^{*}\left(v_{3}\right)=\left( \pm \bar{x}_{3}, \bar{y}_{3}\right)=$ $\left(x_{3}, y_{3}\right)$ so $x_{3}$ is either real or imaginary and $y_{3}$ is real. If $x_{3}$ is real then we have $q^{*}\left(v_{j}\right)=\left(\bar{x}_{j}, \bar{y}_{j}\right)=\left(x_{j}, y_{j}\right)$ for all $v_{j} \in V$ so $q$ is real. If $x_{3}$ is imaginary then $q^{*}\left(v_{j}\right)=\left(-\bar{x}_{j}, \bar{y}_{j}\right)=\left(x_{j}, y_{j}\right)$ so $q\left(v_{j}\right)=\left(x_{j}, y_{j}\right)$ where $x_{j}$ is imaginary and $y_{j}$ is real for all $v_{j} \in V$.

We next consider the the case when $y_{2}$ is imaginary. We have $q^{*}\left(v_{3}\right)=$ $\left( \pm \bar{x}_{3},-\bar{y}_{3}\right)=\left(x_{3}, y_{3}\right)$ so $x_{3}$ is either real or imaginary and $y_{3}$ is imaginary. If $x_{3}$ is imaginary then we have $q^{*}\left(v_{j}\right)=\left(-\bar{x}_{j},-\bar{y}_{j}\right)=\left(x_{j}, y_{j}\right)$ for all $v_{j} \in V$ so $q$ is imaginary. This is impossible since $(G, q)$ is equivalent to $(G, p)$ and so we must have $d(q(u)-q(v))>0$ for all $u v \in E$. If $x_{3}$ is real then $q^{*}\left(v_{j}\right)=\left(\bar{x}_{j},-\bar{y}_{j}\right)=\left(x_{j}, y_{j}\right)$ so $q\left(v_{j}\right)=\left(x_{j}, y_{j}\right)$ where $x_{j}$ is real and $y_{j}$ is imaginary for all $v_{j} \in V$.

In summary $\left(G, q^{*}\right)$ is equal to $(G, q)$ if and only if $q$ is real, or we have $q\left(v_{j}\right)=\left(x_{j}, i y_{j}\right)$ where $x_{j}, y_{j} \in \mathbb{R}$ for all $v_{j} \in V$, or we have $q\left(v_{j}\right)=\left(i x_{j}, y_{j}\right)$ where $x_{j}, y_{j} \in \mathbb{R}$ for all $v_{j} \in V$. We will refer to the latter two such realisations as Minkowski realisations. ${ }^{4}$ It follows that the number of realisations in $S$ which are neither real nor Minkowski must be even. As noted above, the number of real realisations is also even. Thus it is the number of Minkowski realisations which can be odd.

Although the answer to Thurston's question is negative, it would still be of interest to find special families of graphs $G$ for which $r(G, p)=c(G)$ for some generic real realisation $(G, p)$. For example Theorem 7.4 and [17, Theorem 8.2] show that the family of $\mathcal{M}$-connected graphs have this property, and indeed show that $r(G, p)=c(G)=2^{b(G)}$ for all generic real realisations when $G$ is $\mathcal{M}$-connected. Theorems 7.1 and 7.3 show that the family $\mathcal{Q S}$ also has this property.

Acknowledgement We would like to thank Shaun Bullett, Peter Cameron, and Bob Connelly for helpful conversations, and the Fields Institute for support during its 2011 thematic programme on Discrete Geometry and Applications.

\footnotetext{
${ }^{4}$ We can associate such realisations $q$ with realisations $\tilde{q}\left(v_{j}\right)=\left(x_{j}, y_{j}\right)$ in 2-dimensional Minkowski space where distance is given by the Minkowski norm $d(x, y)=\left|-x^{2}+y^{2}\right|$. Results on generic global rigidity in $d$-dimensional Minkowski and other Pseudo-Euclidean spaces are given in [13].
} 


\section{A Proofs of Lemmas 3.1, 3.2, 3.3 and 3.4}

Proof of Lemma 3.1 We first show that $(G, p)$ is congruent to a framework $(G, q)$ in canonical position. As a first step, we define $(G, \tilde{q})$ by putting $\tilde{q}\left(v_{i}\right)=p\left(v_{i}\right)-p\left(v_{1}\right)$ for all $v_{i} \in V$. Then $(G, \tilde{q})$ is congruent to $(G, p)$ and $\tilde{q}\left(v_{1}\right)=(0,0)$. Since $d\left(p\left(v_{2}\right)-p\left(v_{1}\right)\right) \neq 0$, there exists a unique $b_{2} \in \mathbb{C} \backslash\{0\}$ such that $d\left(p\left(v_{2}\right)-p\left(v_{1}\right)\right)=b_{2}^{2}$ and $\operatorname{Arg} b_{2} \in(0, \pi]$. Let $\tilde{q}\left(v_{2}\right)=(a, b)$. Then $a^{2}+b^{2}=d\left(\tilde{q}\left(v_{2}\right)-\tilde{q}\left(v_{1}\right)\right)=d\left(p\left(v_{2}\right)-p\left(v_{1}\right)\right)=b_{2}^{2}$. Put $z_{1}=b / b_{2}$ and $z_{2}=-a / b_{2}$. Then $z_{1}^{2}+z_{2}^{2}=1$ and we may define a realisation $\left(G, q^{*}\right)$ which is congruent to $(G, p)$ and satisfies $q^{*}\left(v_{1}\right)=\tilde{q}\left(v_{1}\right)=(0,0)$ and $q^{*}\left(v_{2}\right)=\left(0, b_{2}\right)$ by putting $q^{*}\left(v_{i}\right)=\left(\begin{array}{rr}z_{1} & z_{2} \\ -z_{2} & z_{1}\end{array}\right) \tilde{q}\left(v_{i}\right)$ for all $v_{i} \in V$. Let $q^{*}\left(v_{3}\right)=\left(a_{3}, b_{3}\right)$. If $a_{3}=0$ or $\operatorname{Arg} a_{3} \in(0, \pi]$, we put $q=q^{*}$. Otherwise $\operatorname{Arg} a_{3} \in(-\pi, 0]$ and we put $q\left(v_{i}\right)=\left(\begin{array}{rr}1 & 0 \\ 0 & -1\end{array}\right) q^{*}\left(v_{i}\right)$ for all $v_{i} \in V$.

It remains to show that $(G, q)$ is unique. We have already seen that $b_{2}$ is uniquely determined by $p$. Choose $d_{1}, d_{2} \in \mathbb{C}$ such that $d\left(p\left(v_{1}\right)-p\left(v_{3}\right)\right)=d_{1}$ and $d\left(p\left(v_{2}\right)-p\left(v_{3}\right)\right)=d_{2}$. Since $(G, p)$ and $(G, q)$ are congruent, we have $a_{3}^{2}+b_{3}^{2}=d_{1}$ and $a_{3}^{2}+\left(b_{3}-b_{2}\right)^{2}=d_{2}$. These equations imply that $b_{3}$ and $a_{3}^{2}$ are uniquely determined by $p$. Since we also have $a_{3}=0$ or $\operatorname{Arg} a_{3} \in(0, \pi]$, $q\left(v_{3}\right)=\left(a_{3}, b_{3}\right)$ is uniquely determined by $p$. By applying a similar argument to $v_{i}$ for all $4 \leq i \leq n$, we have $q\left(v_{i}\right)=\left( \pm a_{i}, b_{i}\right)$ for some fixed $a_{i}, b_{i} \in \mathbb{C}$ which are uniquely determined by $p$. Furthermore, the facts that $(G, q)$ is congruent to $(G, p)$ and $d\left(a_{3}-a_{i}, b_{3}-b_{i}\right) \neq d\left(a_{3}+a_{i}, b_{3}-b_{i}\right)$ whenever $a_{i} \neq 0$, imply that $q\left(v_{i}\right)$ is also uniquely determined by $p$. Hence $(G, q)$ is unique.

Parts (a) and (b) of the lemma follow by noting that $(G, p)$ is collinear if and only if $q\left(v_{i}\right)=\left(0, b_{i}\right)$ for all $v_{i} \in V \backslash\left\{v_{1}, v_{2}\right\}$. Hence we can choose $v_{3}=v_{i}$ with $q\left(v_{3}\right)=\left(a_{3}, b_{3}\right)$ and $a_{3} \neq 0$ if and only if $(G, p)$ is not collinear. The assertions now follow since we have two choices for Arg $b_{2}$, and an additional two choices for $\operatorname{Arg} a_{3}$ when $a_{3} \neq 0$.

\section{Proof of Lemma 3.2}

(a) Relabelling if necessary, we may suppose that the first $m$ columns of $\left.d f\right|_{p}$ are linearly independent. Let $p=\left(p_{1}, p_{2}, \ldots, p_{n}\right)$. Define $h: \mathbb{C}^{m} \rightarrow$ $\mathbb{C}^{m}$ by $h\left(x_{1}, x_{2}, \ldots, x_{m}\right)=f\left(x_{1}, x_{2}, \ldots, x_{m}, p_{m+1}, \ldots, p_{n}\right)$. and let $p^{\prime}=$ $\left(p_{1}, p_{2}, \ldots, p_{m}\right)$. Then $h\left(p^{\prime}\right)=f(p)$ and rank $\left.d h\right|_{p^{\prime}}=m$.

Let $h\left(p^{\prime}\right)=\left(\beta_{1}, \beta_{2}, \ldots, \beta_{m}\right)$. Suppose that $g\left(\beta_{1}, \beta_{2}, \ldots, \beta_{m}\right)=0$ for some 
polynomial $g$ with integer coefficients. Then $g\left(f_{1}(p), f_{2}(p), \ldots, f_{m}(p)\right)=0$. Since $p$ is generic, we have $g(h(x))=0$ for all $x \in \mathbb{C}^{m}$. By the inverse function theorem $h$ maps a sufficiently small open neighbourhood $U$ of $p^{\prime}$ bijectively onto $h(U)$. Thus, for each $y \in h(U)$, there exists $x \in U$ such that $h(x)=y$. This implies that $g(y)=g(h(x))=0$ for each $y \in h(U)$. Since $g$ is a polynomial map and $h(U)$ is an open subset of $\mathbb{C}^{m}$, we have $g \equiv 0$. Hence $h\left(p^{\prime}\right)=f(p)$ is generic.

(b) Since $f_{i}$ is a polynomial with rational coefficients, we have $f_{i}(p) \in \mathbb{Q}(p)$ for all $1 \leq i \leq m$. Thus $\mathbb{Q}(f(p)) \subseteq \mathbb{Q}(p)$. Since $\operatorname{td}[\mathbb{Q}(f(p)): \mathbb{Q}]=n$, we have $\operatorname{td}[\mathbb{Q}(p): \mathbb{Q}]=n$. Thus $p$ is generic, $\overline{\mathbb{Q}(f(p))} \subseteq \overline{\mathbb{Q}(p)}$ and $\operatorname{td}[\overline{\mathbb{Q}(f(p))}: \mathbb{Q}]=$ $n=\operatorname{td}[\overline{\mathbb{Q}(p)}: \mathbb{Q}]$. Suppose $\overline{\mathbb{Q}(f(p))} \neq \overline{\mathbb{Q}(p)}$ and choose $\gamma \in \overline{\mathbb{Q}(p)}-\overline{\mathbb{Q}(f(p))}$. Then $\gamma$ is not algebraic over $\mathbb{Q}(f(p))$ so $\operatorname{td}[\mathbb{Q}(\gamma, f(p)): \mathbb{Q}]=n+1$. This contradicts the facts that $\mathbb{Q}(\gamma, f(p)) \subseteq \overline{\mathbb{Q}(p)}$ and $\operatorname{td}[\overline{\mathbb{Q}(p)}: \mathbb{Q}]=n$. Hence $\overline{\mathbb{Q}(p)}=\overline{\mathbb{Q}(f(p))}$.

(c) Reordering the components of $f$ if necessary, we may suppose that the first $n$ rows of $\left.d f\right|_{p}$ are linearly independent. Let $g: \mathbb{C}^{n} \rightarrow \mathbb{C}^{n}$ by $g(p)=$ $\left(f_{1}(p), f_{2}(p), \ldots, f_{n}(p)\right)$. Then rank $\left.d g\right|_{p}=n$. Since $p$ is generic, we can use (a) to deduce that $g(p)$ is a generic point in $\mathbb{C}^{n}$. We can now use (b) to deduce that $\overline{\mathbb{Q}(p)}=\overline{\mathbb{Q}(g(p))}=\overline{\mathbb{Q}(f(p))}$. It also implies that $g^{-1}(g(p))$ is a zerodimensional algebraic variety and hence is finite. Since $W(p) \subseteq g^{-1}(g(p))$, $W(p)$ is also finite. The fact that $|W(p)|=|W(q)|$ for all generic $q \in \mathbb{C}^{n}$ now follows from the fact that $\mathbb{C}$ is algebraically closed.

Proof of Lemma 3.3 Let $I$ be the ideal of $K(D)[X]$ generated by $\left\{f_{i}(X, D)\right.$ : $1 \leq i \leq m\}$. For each $d \in \mathbb{C}^{t}$ with $\operatorname{td}[K(d): K]=t$ let $I_{d}$ be the ideal of $K(d)[X]$ generated by $\left\{f_{i}(X, d): 1 \leq i \leq m\right\}$. There is an isomorphism from $K(D)(X)$ to $K(d)(X)$ which maps $I$ onto $I_{d}$. Furthermore, Hilbert's Weak Nullstellensatz tells us that $V_{d} \neq \emptyset$ if and only if $I_{d}$ contains a non-zero element of $K(d)$. We may use the above isomorphism to deduce that $V_{d} \neq \emptyset$ if and only if $I$ contains a non-zero element of $K(D)$. The lemma now follows since the latter condition is independent of the choice of $d$.

Proof of Lemma 3.4 The assertion that $d\left(p\left(v_{i}\right)-p\left(v_{j}\right)\right) \neq 0$ for all $1 \leq$ $i<j \leq n$ follows from the hypothesis that $(G, p)$ is quasi-generic. Lemma 3.1 now allows us to assume, without loss of generality, that $p\left(v_{1}\right)=(0,0)$ and $p\left(v_{2}\right)=\left(0, b_{2}\right)$ for some $b_{2} \in \mathbb{C}$.

Let $H$ be a spanning subgraph of $G$ whose edge set corresponds to a max- 
imal set of linearly independent rows of $R(G, p)$. Let $f_{H}: \mathbb{C}^{2 n-3} \rightarrow \mathbb{C}^{|E(H)|}$ be defined by putting $f_{H}(z)$ equal to $d_{H}(0,0,0, z)$ for all $z \in \mathbb{C}^{2 n-3}$. Let $\hat{p}$ be the projection of $p$ onto its last $2 n-3$ coordinates. Then rank $\left.d f_{H}\right|_{\hat{p}}=$ $\operatorname{rank} R(H, p)=|E(H)|$ so Lemma $3.2(\mathrm{a})$ implies that $f_{H}(\hat{p})=d_{H}(p)$ is generic.

Suppose $G$ is rigid. Then $|E(H)|=2 n-3$, and Lemma 3.2(b) gives $\overline{\mathbb{Q}(p)}=\overline{\mathbb{Q}(\hat{p})}=\overline{\mathbb{Q}\left(f_{H}(\hat{p})\right)}=\overline{\mathbb{Q}\left(d_{H}(p)\right)}$. Since $\mathbb{Q}\left(d_{H}(p)\right) \subseteq \mathbb{Q}\left(d_{G}(p) \subseteq \mathbb{Q}(p)\right.$, we have $\overline{\mathbb{Q}(p)}=\overline{\mathbb{Q}\left(d_{G}(p)\right)}$ and

$$
\operatorname{td}\left[\mathbb{Q}\left(d_{G}(p)\right): \mathbb{Q}\right]=\operatorname{td}\left[\mathbb{Q}\left(d_{H}(p)\right): \mathbb{Q}\right]=|E(H)|=\operatorname{rank} R(G, p) .
$$

It remains to consider the case when $G$ is not rigid. Let $G_{1}, G_{2}, \ldots, G_{t}$ be the maximal rigid subgraphs of $G$. By the previous paragraph, $\operatorname{td}\left[\mathbb{Q}\left(d_{G_{i}}(p)\right)\right.$ : $\mathbb{Q}]=2\left|V\left(G_{i}\right)\right|-3$ for all $1 \leq i \leq t$, and by a standard result on 2-dimensional rigidity, rank $R(G, p)=\sum_{i=1}^{t} 2\left|V\left(G_{i}\right)\right|-3$. Hence

$\operatorname{td}\left[\mathbb{Q}\left(d_{G}(p)\right): \mathbb{Q}\right] \leq \sum_{i=1}^{t} \operatorname{td}\left[\mathbb{Q}\left(d_{G_{i}}(p)\right): \mathbb{Q}\right]=\sum_{i=1}^{t}\left(2\left|V\left(G_{i}\right)\right|-3\right)=\operatorname{rank} R(G, p)$.

On the other hand, we may use the fact that $d_{H}(p)$ is generic to deduce that $\operatorname{td}\left[\mathbb{Q}\left(d_{G}(p)\right): \mathbb{Q}\right] \geq \operatorname{td}\left[\mathbb{Q}\left(d_{H}(p)\right): \mathbb{Q}\right]=|E(H)|=\operatorname{rank} R(G, p)$. Thus $\operatorname{td}\left[\mathbb{Q}\left(d_{G}(p)\right): \mathbb{Q}\right]=\operatorname{rank} R(G, p)$.

\section{References}

[1] L. Asimow And B. Roth, Rigidity of graphs II, J. Math. Anal. Appl. 68 (1979), 171-190.

[2] A.R. Berg And T. Jordán, A proof of Connelly's conjecture on 3-connected circuits of the rigidity matroid, J. Combinatorial Theory, Ser. B. 88 (2003), 77-97.

[3] A.R. Berg And T. Jordán, Algorithms for graph rigidity and scene analysis, Proc. 11th Annual European Symposium on Algorithms (ESA) 2003, (G. Di Battista, U. Zwick, eds) Springer Lecture Notes in Computer Science 2832 (2003), 78-89. 
[4] C. Borcea And I. Streinu, The number of embeddings of minimally rigid graphs, Discrete Comput Geom 31 (2004), 287-303.

[5] J. Capco, M. Gallet, G. Grassegger, C. Koutschan, N. LubBes AND J. Schicho, The number of realizations of a Laman graph, preprint arXiv 1701.05500v1.

[6] R. Connelly, Generic global rigidity, Disc. Comput. Geom. 33 (2005), 549-563.

[7] I.Z. Emiris And G. Moroz, The assembly modes of 11-bar linkages, in Proc. IFToMM World Cong. Mechanism and Machine Sc. Guanajuato, Mexico (2011).

[8] I.Z. Emiris And I. D. Psarros, Counting Euclidean embeddings of rigid graphs, preprint arXiv:1402.1484v1.

[9] I.Z. Emiris, E.P. Tsigaridas and A. Varvitsiotis, Mixed volume and distance geometry techniques for counting Euclidean embeddings of rigid graphs, in Distance geometry: Theory, Methods, and Appications Springer, New York 2013 23-45.

[10] Z. Fekete, T. Jordán And W. Whiteley, An Inductive Construction for Plane Laman Graphs via Vertex Splitting, Algorithms - ESA 2004, Lecture Notes in Comput. Sci., 3221 (2004), 299-310.

[11] X.S.Gao And Shang-Ching Chou, Solving geometric constraint systems, II. A symbolic approach and decision of rc-constructability, Computer Aided Design, 30 (1998), 115-122.

[12] S. Gortler, A. Healy, and D. Thurston, Characterizing generic global rigidity, American J. Math. 132 (2010) 897-939.

[13] S. Gortler And D. Thurston, Generic global rigidity in complex and pseudo-euclidean spaces, in Rigidity and Symmetry, Fields Institute Communications 70, Springer New York 2014 131-154.

[14] B. Hendrickson, Conditions for unique graph realisations, SIAM J. Comput. 21 (1992), 65-84.

[15] W.V.D.Hodge And D.Pedoe, Methods of Algebraic Geometry Volume 2, Cambridge University Press, 1952. 
[16] B. JaCkson And T. Jordán, Connected rigidity matroids and unique realisations of graphs, J. Combin. Theory Ser. B 94 (2005), 1-29.

[17] B. Jackson, T. Jordán, and Z. Szabadka, Globally linked pairs of vertices in equivalent realizations of graphs, Discrete and Computational Geometry 35 (2006), 493-512.

[18] B. Jackson And J. C. Owen, The number of equivalent realisations of a rigid graph, preprint arXiv 1204.1228v2.

[19] B. JaCkson And J. C. Owen, Equivalent realisations of rigid graphs, in Proc. 10th Japanese-Hungarian Sym. Disc. Math. App. (A. Frank, A. Recski and G. Wiener eds.) Budapest 2017, 283-289.

[20] G. Laman, On graphs and rigidity of plane skeletal structures, J. Engrg. Math 4 (1970), 331-340.

[21] L. Liberti, C. Lavor, J. Alencar, G. Abud, Counting the number of solutions of ${ }^{K} D M D G P$ instances, in GSI 2013, Lecture Notes in Comp. Sci. 8085 (2013), 224-230.

[22] L. Liberti, C. Lavor, A. Mucherino, The discretizable molecular distance problem seems easier on proteins, in Distance Geometry: Theory Methods and Applications A. Mucherino et al. eds. 2013, 47-60.

[23] L. Liberti, B. Masson, J.Lee, C. Lavor, A. Mucherino, On the number of realisations of certain Henneberg graphs arising in protein conformation, Disc. Appl. Math 165 (2014), 213-232.

[24] L. Lovász And Y. Yemini, On generic rigidity in the plane, SIAM J. Algebraic Discrete Methods 3 (1982), 91-98.

[25] V.-H. NGuyen, 1-extensions and global rigidity of generic directionlength frameworks, Internat. J. Comput. Geom. Appl., 22 (2012), 577591.

[26] J.C. Owen, Algebraic solution for geometry from dimensional constraints, in ACM Symposium on Foundations in Solid Modeling, Austin, Texas 1991, 397-407. 
[27] J. C. Owen And S. C. Power, The Non-Solvability by Radicals of Generic 3-Connected Planar Laman Graphs, Transactions of the American Mathematical Society 359 (2007), 2269-2303.

[28] J.G. Oxley, Matroid theory, Oxford Science Publications. The Clarendon Press, Oxford University Press, New York, 1992.

[29] I. Stewart, Galois Theory, 3rd ed., Chapman and Hall/CRC Mathematics, 2004.

[30] J.B. SAXE, Embeddability of weighted graphs in $k$-space is strongly NPhard, Tech. Report, Computer Science Department, Carnegie-Mellon University, Pittsburgh, PA, 1979.

[31] Z. SzaBADKa, Globally rigid frameworks and rigid tensegrity graphs in the plane, Ph.D. thesis, Eötvös Loránd University, Institue of Mathematics, 2010.

[32] A.J.Sommese And C.W.Wampler, II, The Numerical Solution of Systems of Polynomials, World Scientific, 2005.

[33] R. Steffens and T. Theobald, Mixed volume techniques for embeddings of Laman graphs, Comp. Geom.: Theo. Appl. 43 (2010)84-93.

[34] W. Whiteley Vertex splitting in isostatic frameworks, Structural Topology 16 (1990), 23-30

[35] W. Whiteley, Some matroids from discrete applied geometry, in $M a-$ troid theory, Contemp. Math., 197, Amer. Math. Soc., Providence, RI, 1996, 171-311. 